\title{
Split Special Lagrangian Geometry
}

\author{
F. Reese Harvey and H. Blaine Lawson, Jr.*
}

\begin{abstract}
One purpose of this article is to draw attention to the seminal work of J. Mealy in 1989 on calibrations in semi-riemannian geometry where split SLAG geometry was first introduced. The natural setting is provided by doing geometry with the complex numbers $\mathbf{C}$ replaced by the double numbers $\mathbf{D}$, where $i$ with $i^{2}=-1$ is replaced by $\tau$ with $\tau^{2}=1$. A rather surprising amount of complex geometry carries over, almost untouched, and this has been the subject of many papers. We briefly review this material and, in particular, we discuss Hermitian D-manifolds with trivial canonical bundle, which provide the background space for the geometry of split SLAG submanifolds.

A removable singularities result is proved for split SLAG subvarieties. It implies, in particular, that there exist no split SLAG cones, smooth outside the origin, other than planes. This is in sharp contrast to the complex case.

Parallel to the complex case, space-like Lagrangian submanifolds are stationary if and only if they are $\theta$-split SLAG for some phase angle $\theta$, and infinitesimal deformations of split SLAG submanifolds are characterized by harmonic 1-forms on the submanifold.

We also briefly review the recent work of Kim, McCann and Warren who have shown that split Special Lagrangian geometry is directly related to the Monge-Kantorovich mass transport problem.
\end{abstract}

*Partially supported by the N.S.F. 


\section{TABLE OF CONTENTS}

1. Introduction.

2. Double Numbers and Double Holomorphic Functions.

3. Double $n$-Space.

4. The Special Lagrangian Calibration.

5. Split SLAG Submanifolds.

6. Split SLAG Graphs in $\mathrm{D}^{n}$

7. Removable Singularities for split SLAG Varieties.

8. Double Manifolds.

9. Hermitian D-Manifolds.

10. Kähler D-Manifolds.

11. Ricci-Flat Kähler D-Manifolds.

12. Split SLAG Submanifolds in the General Setting.

13. Deformations and the McLean Theorem in the Split Case

14. Relation to the Mass Transport Problem - Work of Kim, McCann and Warren

15. Further Work of Mealy.

16. Lagrangian Submanifolds of Constant Phase and Volume Maximization

\section{Appendices:}
A. A Canonical Form for Space-like $n$-Planes in $\mathrm{D}^{n}$.
B. Projective and Hermitian Projective Varieties over D.
C. Degenerate Projections.
D. Singularities and Semi-Riemannian Calibrations.
E. Singularities in Split SLAG Geometry.
F. Split SLAG Singularities in Dimension Two. 


\section{Introduction.}

In an interesting recent series of papers $[\mathrm{KM}],\left[\mathrm{W}_{1}\right],[\mathrm{KMW}]$ Y.-H. Kim, R. McCann and M. Warren established a direct relationship between the classical Monge-Kantorovich mass transport problem and split special Lagrangian geometry. This geometry is one of the basic calibrated semi-riemannian geometries introduced by Jack Mealy in his seminal work $\left[\mathrm{M}_{1,2}\right]$ in 1989-90. The ambient spaces are analogues of complex Kähler manifolds for which the literature is vast and not unified $^{1}$ - they are sometimes called paraKähler and sometimes Bi-Lagrangian (we shall call them Káhler D-manifolds). Despite the widespread interest, Mealy's results about calibrations on such manifolds seem to have gone unnoticed. One purpose of this article is to give a brief self-contained discussion of $\mathbf{D}$-manifolds so that we can discuss split special Lagrangian geometry and present the work of Mealy in this important case. We shall then explain how the work of Kim-McCann-Warren fits into the picture. Along the way we will also establish some new results concerning split special Lagrangian subvarieties. One is a removable-singularities result which implies, in particular, that any split SLAG cone, which is smooth and connected outside the origin, is a plane (in sharp contrast to the classical non-split case where such cones exist in abundance). Another can be interpreted as the strongest possible regularity result for $d$-closed split special Lagrangian rectifiable currents in dimension 2 .

Throughout the exposition we emphasize the two distinct approaches to $\mathbf{D}$-manifolds. The first is in strong analogy with complex geometry, where the double numbers $\mathbf{D}$ (see $\S 2$ ) are considered as a replacement for the complex numbers $\mathbf{C}$, and a surprising number of definitions and results carry over. In the second viewpoint the double numbers are viewed as the algebra $\mathbf{D}=\mathbf{R} \oplus \mathbf{R}$. Here one focuses on the canonically defined pair of transverse $n$-dimensional foliations which give rise locally to null coordinates. In this setting many classical objects, such as holomorphic $n$-forms, which are written as usual in complex notation, appear quite differently in null coordinates. A striking example is the equation for the (split) special Lagrangian potential function. In "complex coordinates" the equation resembles that of the non-split case - all the odd elementary symmetric functions of the hessian must sum to zero. In null coordinates, it becomes the Monge-Ampère equation $\operatorname{det} \operatorname{Hess} u=1$.

The algebra $\mathbf{D}$ mentioned in the paragraph above has at least four names: the double numbers, the para-complex numbers, the Lorentz numbers, and the hyperbolic numbers. It is the algebra over $\mathbf{R}$ generated by 1 and $\tau$ where $\tau^{2}=1$. In other words $\mathbf{D}$ is the Clifford algebra for the negative definite quadratic form on $\mathbf{R}$ (while $\mathbf{C}$ comes from the positive definite one). The algebras $\mathbf{C}$ and $\mathbf{D}$ are the only commutative normed algebras aside from $\mathbf{R}$. They are like twin sisters living in parallel universes, one elliptic and the other hyperbolic. Writing elements in $\mathbf{D}$ as $z=$ $x+\tau y$, and setting $\bar{z}=x-\tau y$ one can proceed formally to define $\mathbf{D}$ holomorphic functions (of one and several variables), and the analogues of complex manifolds (called D-manifolds here), holomorphic vector bundles, the Dolbeault complex, and much more. There are $\mathbf{D}$ projective spaces and grassmannians. In fact every real algebraic variety has an associated $\mathbf{D}$-variety via base change. Even more striking are the metric analogues. There are hermitian D-manifolds, Kähler D-manifolds and Calabi-Yau D-manifolds. Many basic theorems carry over. This is discussed below.

However, as the reader may have noticed, after setting $e=\frac{1}{2}(1+\tau)$ and $\bar{e}=\frac{1}{2}(1-\tau), \mathbf{D}$ becomes the double algebra $\mathbf{R} \oplus \mathbf{R}$ of elements $u e+v \bar{e}$ where $u, v \in \mathbf{R}$ (and $e^{2}=e, \bar{e}^{2}=\bar{e}$ and $e \bar{e}=0)$. These are the null-coordinates mentioned above. The analogues of many fundamental objects look quite different in these coordinates. For example, $-\tau \partial \bar{\partial}=d_{u} d_{v}$ where $d_{u}$ and $d_{v}$ are the standard deRham differentials in the variables $u$ and $v$.

${ }^{1}$ For example, see $[\mathrm{AMT}],[\mathrm{CFG}],[\mathrm{EST}],[\mathrm{GM}],[\mathrm{GGM}],[\mathrm{IZ}],[\mathrm{SS}]$ and references cited therein. 
The paper is organized as follows. After a brief introduction to analysis over $\mathbf{D}$ we examine the split special Lagrangian calibration $\Phi=\operatorname{Re} d z$ in $\mathbf{D}^{n}$ and prove Mealy's sharp inequality which states that $\Phi$ is a (reverse) calibration which is minimized on space-like special Lagrangian $n$ planes. By the fundamental lemma of calibrations this implies that split SLAG submanifolds are homologically volume-maximizing. This is done in $\S \S 1-5$.

Recall that the natural exterior differential system (EDS) for SLAG submanifolds of $\mathbf{C}^{n}$ is generated by $\operatorname{Im} d z$ and the standard symplectic (Kähler) form $\omega$. A submanifold is an integral submanifold for this EDS if and only if it is SLAG when correctly oriented. The parallel to this in the split case (Theorem 5.2) is almost identical. Again the differential system is generated by $\operatorname{Im} d z$ and $\omega$ (this time on $\mathbf{D}^{n}$ ), but an integral submanifold is split SLAG modulo orientation if and only if it is space-like. These more general integral submanifolds, which are not necessarily space-like, will be called unconstrained split SLAG submanifolds

In $\S 6$ we examine split SLAG submanifolds $M$ which are graphs of mappings. If $M$ is the oriented graph of $F: \Omega \rightarrow \operatorname{Im} \mathbf{D}^{n}$ where $\Omega$ is a simply-connected domain in $\operatorname{Re} \mathbf{D}^{n} \cong \mathbf{R}^{n}$, then following Mealy, $F=d f$ for a potential function $f$ for which

$$
\operatorname{Im} \operatorname{det}_{\mathbf{D}}(I+\tau \operatorname{Hess} f)=0 \quad \text { and } \quad-I<\operatorname{Hess} f<I .
$$

If on the other hand, $M$ similarly is expressed as the graph $v=G(u)$ over a domain in null coordinates, then $G=d g$ for a potential function $g$ satisfying (cf. [Hi $\left.{ }_{1}\right]$ )

$$
\operatorname{det} \operatorname{Hess} g=1 \quad \text { and } \quad \text { Hess } g>0 \text {. }
$$

In $\S 7$ we prove the following removable-singularities result.

THEOREM 7.1. Let $\Omega \subset \mathbf{R}^{n}$ be a convex domain in $\mathbf{R}^{n}$ and consider the "tube" domain $\Omega \times \tau \mathbf{R}^{n} \subset \mathbf{R}^{n} \oplus \tau \mathbf{R}^{n}=\mathbf{D}^{n}$. Let $\Sigma \subset \Omega$ be a compact subset of Hausdorff ( $\left.n-2\right)$-measure zero. Then any closed, connected split SLAG submanifold

$$
M \subset(\Omega-\Sigma) \times \tau \mathbf{R}^{n}
$$

has closure in $\Omega \times \tau \mathbf{R}^{n}$ which is the graph of a real analytic mapping $F: \Omega \rightarrow \mathbf{R}^{n}$. Furthermore, $F=d f$ where $f: \Omega \rightarrow \mathbf{R}$ satisfies (1.1).

Corollary 7.2. (Absence of Cones). Suppose $C \subset \mathbf{D}^{n}$ is a split SLAG cone which is regular and connected outside the origin. Then $C$ is an $n$-plane.

This is in sharp contrast to the non-split case where SLAG-cones exist in all dimensions and there are many SLAG-varieties with singularities of high codimension (cf. $\left[\mathrm{HL}_{1}\right],\left[\mathrm{J}_{*}\right],\left[\mathrm{H}_{*}\right],\left[\mathrm{HK}_{*}\right]$ and the references therein).

The proof of Theorem 7.1 relies on playing off the two different coordinate pictures for $z \in \mathbf{D}^{n}$. With $z=x+\tau y$ we show that the projection of $M$ to $\Omega-\Sigma$ must be a covering map. This leads to a potential with a Lipschitz extension across $\Sigma$. We then rotate to null coordinates $z=u e+v \bar{e}$ (the Cayley transform) and apply deep results of Caffarelli $\left[\mathrm{Ca}_{*}\right]$ to get full regularity.

Corollary 7.2 would suggest that singularities of codimension $\geq 3$ do not exist on split SLAG subvarieties. By contrast, singularities of codimension-2 certainly do exist in all dimensions. It turns out that when $n=2$ the exterior differential system for (unconstrained) split SLAG submanifolds is equivalent to the Cauchy-Riemann system for complex curves. Specifically we have the following. 
THEOREM F.3. There exists a permutation of real coordinates $\mathbf{C}^{2} \leftrightarrow \mathbf{D}^{2}$, such that:

(a) holomorphic chains are transformed to unconstrained split SLAG currents and vice-versa.

(b) holomorphic chains satisfing a $45^{\circ}$-rule are transformed to split SLAG currents and vice-versa.

Since complex curves have local uniformizing parameters, this can be interpreted as a very strong regularity result for split SLAG subvarieties when $n=2$. (A split SLAG subvariety is a $d$-closed rectifiable current $T$ whose tangent planes $\vec{T}$ are space-like special Lagrangian almost everywhere. See Appendices D and E for a fuller discussion.) Theorem F.3 follows from work of the first author and B. Shiffman $[\mathrm{HS}],[\mathrm{S}]$ which states that: a $d$-closed rectifiable 2-current defined in an open subset $X$ of $\mathbf{C}^{2}$, whose (unoriented) tangent space $\vec{T}$ is a complex line $\|T\|$-a.e., is a $T$ holomorphic chain in $X$. That is, $T$ is a locally finite sum $T=\sum_{j} n_{j}\left[V_{j}\right]$ where for each $j, n_{j} \in \mathbf{Z}$ and $V_{j}$ is a 1-dimensional complex analytic subvariety of $X$.

This result also clearly implies that isolated singularities do occur on split SLAG subvarieties when $n=2$. They are the singular points of the underlying holomorphic curves. Taking products of these with appropriately chosen linear subspaces gives codimension-2 singularities on split SLAG subvarieties in all dimensions $n$.

It is interesting to note that since locally irreducible holomorphic curves have well defined tangent lines at singular points, this procedure does not yield interesting cones. So far, no nonplanar irreducible cones are known.

In sections 8 through 11 we discuss the geometry of $\mathbf{D}$-manifolds. In particular we examine hermitian D-manifolds, Kähler D-manifolds, and Ricci-Flat Kähler D-manifolds. A number of examples are given. In section 12 we discuss split SLAG submanifolds in this general setting. In section 13 we consider the deformation theory for these submanifolds in the Ricci-flat Kähler case. It is shown that the basic results of McLean $\left[\mathrm{Mc}_{1,2}\right]$ for the classical (non-split) special Lagrangian submanifolds carry over to this case (cf. Warren $\left[\mathrm{W}_{2}\right]$ ).

Then in $\S 14$ we discuss the work of Kim-McCann-Warren on the relevance of split special Lagrangian geometry to the mass transport problem.

In section 15 we briefly survey the work of Mealy on other calibrations in geometries of indefinite signature.

In section 16 we analyze the case of Lagrangian submanifolds of $\mathbf{D}^{n}$ (or more generally of Ricci-flat Kähler D-manifolds) on which the restricted metric is everywhere non-degenerate of signature $p, q(p+q=n)$. We show that any such manifold $M$ is stationary (mean curvature zero) if and only if the restriction of $d z$ to $M$ is of constant phase (see [HL $\mathrm{HL}_{1}$, [D] for parallel cases). When $p=n$ (respectively, 0 ), a compact oriented constant-phase submanifold maximizes volume among all oriented space-like (respectively, time-like) submanifolds with the same boundary. For $0<p<n$, volume is not maximized in general, but it is maximized among oriented Lagrangian submanifolds of the same type. In all cases the volume inequality becomes an equality only when the competitor is also of the same constant phase.

Appendix A gives a canonical form for a space-like $n$-planes in $\mathbf{D}^{n}$, under the action of the D-unitary group, which is used in proving Mealy's theorem.

Appendix B treats projective D-subvarieties and their induced split Kähler geometry.

Appendix $\mathrm{C}$ shows that the result proved in $\left[\mathrm{HL}_{1}\right]$ characterizing special Lagrangian submanifolds with degenerate projections, carries over to the split case.

Appendix D presents the general theory of calibrations on semi-riemannian manifolds. This entails, among other things, the establishing of a canonical polar form for space-like currents which 
are representable by integration. The Fundamental Theorem for Semi-Riemannian Calibrations is then established for general $\phi$-subvarieties (rectifiable $\phi$-currents).

Appendix E examines the special case of split SLAG subvarieties which are shown to be volume maximizing (cf. [KMW]).

Appendix $\mathrm{F}$ is devoted to the case $n=2$ which was discussed above.

We would like to thank Luis Caffarelli, Young-Heon Kim and Micah Warren for helpful comments.

\section{Double numbers and Double Holomorphic Functions.}

In this section we recall the basics of double or para-complex analysis. By the double numbers we mean the 2-dimensional algebra $\mathbf{D}$ over $\mathbf{R}$ generated by 1 and $\tau$ with $\tau^{2}=1$. This is the only commutative normed algebra other than the real and complex numbers. In analogy with the complex numbers, each $z \in \mathbf{D}$ can be written as

$$
z=x+\tau y
$$

with $x, y \in \mathbf{R}$ defined to be the real and imaginary parts. However, choosing the basis $e=\frac{1}{2}(1-\tau)$ and $\bar{e}=\frac{1}{2}(1+\tau)$ each $z \in \mathbf{D}$ can be written as

$$
z=u e+v \bar{e}
$$

where $(u, v)$ are called the null-coordinates of $z$. Note that $e^{2}=e, \bar{e}^{2}=\bar{e}$ and $e \bar{e}=0$. Thus we see that $\mathbf{D}$ is just the algebra of diagonal $2 \times 2$-matrices $(u, v)$, i.e., $\mathbf{D}=\mathbf{R} \oplus \mathbf{R}$ as algebras. Note the relations

$$
\tau \cdot e=-e \quad \text { and } \quad \tau \cdot \bar{e}=\bar{e} .
$$

The representation (2.1) leads to a vast development of analysis and geometry in parallel with the complex case. This development is frequently demystified by using the null coordinates (2.2).

Conjugation in $\mathbf{D}$ is defined exactly as in the complex case: $\bar{z}=x-\tau y=v e+u \bar{e}$. However

$$
\langle z, z\rangle=z \bar{z}=x^{2}-y^{2}=u v
$$

defines a quadratic form of signature $(1,1)$. The algebra $\mathbf{D}$ is normed in the sense that $\langle z w, z w\rangle=$ $\langle z, z\rangle\langle w, w\rangle$. The notion of vanishing or being zero in complex analysis is replaced by the notion of being null, i.e., $z \bar{z}=0$, in which case having an inverse is impossible. Otherwise, $z^{-1}=\bar{z} /\langle z, z\rangle$ as in the complex case.

The multiplicative group $\mathbf{D}^{*}$ of non-null numbers has four connected components. Let $\mathbf{D}^{+}$ denote the component containing 1. Exponentiation gives an isomorphism:

$\exp : \mathbf{D} \cong \mathbf{D}^{+}$with inverse $\log : \mathbf{D}^{+} \rightarrow \mathbf{D}$. Note that using $(2.1)$ and $(2.2)$

$$
\begin{aligned}
\exp (z) & =\exp (x)(\cosh y+\tau \sinh y) \\
& =e \exp (u)+\bar{e} \exp (v)
\end{aligned}
$$

The space-like unit sphere has two components parameterized by $\pm \exp (\tau \theta)$.

As in complex analysis we now treat $\mathbf{D}$ as $\mathbf{R}^{2}$ and consider smooth $\mathbf{D}$-valued functions on open sets $U \subset \mathbf{D}$. This leads to several parallels. For example the $\mathbf{D}$-valued 1 -forms

$$
d z=d x+\tau d y=e d u+\bar{e} d v \quad \text { and } \quad d \bar{z}=d x-\tau d y=e d v+\bar{e} d u
$$


have duals

$$
\frac{\partial}{\partial z}=\frac{1}{2}\left(\frac{\partial}{\partial x}+\tau \frac{\partial}{\partial y}\right)=e \frac{\partial}{\partial u}+\bar{e} \frac{\partial}{\partial v} \quad \text { and } \quad \frac{\partial}{\partial \bar{z}}=\frac{1}{2}\left(\frac{\partial}{\partial x}-\tau \frac{\partial}{\partial y}\right)=e \frac{\partial}{\partial v}+\bar{e} \frac{\partial}{\partial u} .
$$

However,

$$
4 \frac{\partial^{2}}{\partial z \partial \bar{z}}=\frac{\partial^{2}}{\partial x^{2}}-\frac{\partial^{2}}{\partial y^{2}}=4 \frac{\partial^{2}}{\partial u \partial v}
$$

is the wave equation, so that the regularity obtained in the complex case is lacking. Nevertheless, we define a smooth function $F: U \rightarrow \mathbf{D}$ to be $\mathbf{D}$-holomorphic if

$$
\frac{\partial F}{\partial \bar{z}}=0
$$

If we write $F=e f+\bar{e} g$ with $f$ and $g$ real-valued, then using null coordinates,

$$
\begin{gathered}
\bar{\partial} F \equiv \frac{\partial F}{\partial \bar{z}} d \bar{z} \quad \text { becomes } \bar{\partial}(e f+\bar{e} g)=e d_{v} f+\bar{e} d_{u} g . \quad \text { Also, } \\
\partial F \equiv \frac{\partial F}{\partial z} d z \quad \text { becomes } \partial(e f+\bar{e} g)=e d_{u} f+\bar{e} d_{v} g .
\end{gathered}
$$

Here $d_{u}$ and $d_{v}$ are the usual exterior differentiation operators in the null coordinates $u$ and $v$. Thus

$$
\begin{gathered}
F=e f+\bar{e} g \text { is } \mathbf{D} \text {-holomorphic } \\
f=f(u) \text { and } g=g(v) \text { are functions of the single variable } u \text { and } v \text { respectively. }
\end{gathered}
$$

For any $F=e f+\bar{e} g$ we have $2 \operatorname{Re} F=f+g$ and $2 \operatorname{Im} F=f-g$. Consequently, we see that the real parts of $\mathbf{D}$-holomorphic functions are functions of the form $f(u)+g(v)$. Observe that just as in the complex case:

$$
F \text { is } \mathbf{D} \text { holomorphic and } \operatorname{Re} F=0 \Rightarrow F=\tau c \text { is constant }
$$

despite the hyperbolic character of (2.4). Finally note that

$$
-\tau \partial \bar{\partial}=d_{u} d_{v}
$$

is a real operator. One can show that any function in the kernel of this operator is locally the real part of a $\mathbf{D}$-holomorphic function.

\section{Double n-Space $\mathrm{D}^{n}$.}

The standard formulas in several complex variables have rather obvious analogues. Define

$$
\partial=\sum_{k=1}^{n} d z_{k} \wedge \frac{\partial}{\partial z_{k}} \quad \text { and } \quad \bar{\partial}=\sum_{k=1}^{n} d \bar{z}_{k} \wedge \frac{\partial}{\partial \bar{z}_{k}}
$$

operating on $\mathbf{D}$-valued differential forms (forms with coefficients in $\mathbf{D}$ at each point). These operators satisfy the usual relations: $\partial^{2}=\bar{\partial}^{2}=\partial \bar{\partial}+\bar{\partial} \partial=0$ and $d=\partial+\bar{\partial}$. We can define the real operator $d^{c}=\tau(\partial-\bar{\partial})=2 \operatorname{Im}(\partial)$ so that $\partial=\frac{1}{2}\left(d+\tau d^{c}\right)$. Then $-\frac{\tau}{2} \partial \bar{\partial}=\frac{1}{4} d d^{c}$ is a real 
operator. So far these are exactly the same (no sign changes) as in the complex case. Switching to null coordinates the operators are given by

$$
\partial=e d_{u}+\bar{e} d_{v} \quad \text { and } \quad \bar{\partial}=e d_{v}+\bar{e} d_{u} .
$$

The standard symplectic form $\omega$ on $\mathbf{R}^{2 n}$ can be written in the two ways $\omega=\sum_{j} d x_{j} \wedge d y_{j}=$ $\frac{1}{2} \sum_{j} d u_{j} \wedge d v_{j}$ and has the $-\frac{1}{2} \tau \partial \bar{\partial}=\frac{1}{2} d_{u} d_{v}$ potential $\varphi=\sum_{j} z_{j} \bar{z}_{j}=\sum_{j} u_{j} \bar{v}_{j}$.

Definition 3.1. A D-valued function $F$ on an open subset of $\mathbf{D}^{n}$ is $\mathbf{D}$-holomorphic if $\bar{\partial} F=0$. Note that in null coordinates $(u, v)$ if we write $F=e f+\bar{e} g$ with $f$ and $g$ real-valued, then

$F$ is D-holomorphic $\Longleftrightarrow d_{v} f=0$ and $d_{u} g=0 \Longleftrightarrow f=f(u)$ and $g=g(v)$.

On $\mathbf{D}^{n}$ we have the standard $\mathbf{D}$-valued hermitian form and real inner product:

$$
(z, w)=\sum_{j=1}^{n} z_{j} \bar{w}_{j} \quad \text { and } \quad\langle z, w\rangle=\operatorname{Re}(z, w)=\operatorname{Re} \sum_{j=1}^{n} z_{j} \bar{w}_{j} .
$$

The associated real quadratic form $\langle z, z\rangle=\sum\left(x_{j}^{2}-y_{j}^{2}\right)$ has split or neutral signature. Note that the standard $\mathbf{D}$-valued hermitian form has imaginary part $-\omega$. Hence, exactly as in the complex case

$$
(\cdot, \cdot)=\langle\cdot, \cdot\rangle-\tau \omega(\cdot, \cdot)
$$

The analogue of the complex structure operator $J$ is the operator $T z=\tau z$ of multiplication by $\tau$ (see (8.3)). Now (3.3) leads to an array of formulas such as: $\left\langle z^{\prime}, T z\right\rangle=\omega\left(z^{\prime}, z\right)$ relating $\langle\cdot, \cdot\rangle, \omega$ and $T$ (see $(9.3))$.

The D-linear maps from $\mathbf{D}^{n}$ to $\mathbf{D}^{m}$ correspond to the set $M_{n, m}(\mathbf{D})$ of $n \times m$-matrices with entries in $\mathbf{D}$. By standard algebra each square matrix $A \in M_{n}(\mathbf{D})$ has a $\mathbf{D}$-determinant with the usual properties such as: $\operatorname{det}_{\mathbf{D}}(A B)=\left(\operatorname{det}_{\mathbf{D}} A\right)\left(\operatorname{det}_{\mathbf{D}} B\right), \operatorname{det}_{\mathbf{D}} A^{t}=\operatorname{det}_{\mathbf{D}} A$, and $A \widetilde{A}^{t}=\left(\operatorname{det}_{\mathbf{D}} A\right) I$ where $\widetilde{A}$ is the cofactor matrix. Thus $A$ has an inverse $A^{-1} \in M_{n}(\mathbf{D})$ if and only if $\operatorname{det}_{\mathbf{D}} A$ has in inverse, i.e., $\operatorname{det}_{\mathbf{D}} A \in \mathbf{D}^{*}$ is non-null. Let $\mathrm{GL}_{n}(\mathbf{D}) \subset M_{n}(\mathbf{D})$ denote the group of invertible elements. Setting $d z \equiv d z_{1} \wedge \cdots \wedge d z_{n}$ we have that if $z^{\prime}=A z$ for $A \in M_{n}(\mathbf{D})$, then $d z^{\prime}=\operatorname{det}_{\mathbf{D}} A d z$. Since $d z \wedge d \bar{z}$ is a non-null real multiple of $d x \wedge d y$, this proves that (as in the complex case)

$$
\left(\operatorname{det}_{\mathbf{D}} A\right)\left(\overline{\operatorname{det}_{\mathbf{D}} A}\right)=\operatorname{det}_{\mathbf{R}} A .
$$

Writing $A=e B+\bar{e} C \in M_{n}(\mathbf{D})$ with $B, C \in M_{n}(\mathbf{R})$ and noting that $d z=e d u+\bar{e} d v$ yields the simple formula

$$
\operatorname{det}_{\mathbf{D}}(e B+\bar{e} C)=e \operatorname{det}_{\mathbf{R}} B+\bar{e} \operatorname{det}_{\mathbf{R}} C
$$

for the D-determinant. In particular, $\operatorname{det}_{\mathbf{D}} A \in \mathbf{D}^{+} \Longleftrightarrow \operatorname{det}_{\mathbf{R}} B>0$ and $\operatorname{det}_{\mathbf{R}} C>0$.

Each real linear map from $\mathbf{D}^{n}$ to $\mathbf{D}^{m}$ decomposes into the sum of a $\mathbf{D}$-linear map and an anti-D-linear map. Thus the Jacobian of a smooth mapping $F$ from (an open subset of) $\mathbf{D}^{n}$ to $\mathbf{D}^{m}$ decomposes as

$$
J_{\mathbf{R}}(F)=J_{\mathbf{D}}^{1,0}(F)+J_{\mathbf{D}}^{0,1}(F) \equiv \frac{\partial F}{\partial z}+\frac{\partial F}{\partial \bar{z}}
$$

and $F$ is said to be $\mathbf{D}$-holomorphic if the anti-linear part $J_{\mathbf{D}}^{0,1}(F)=0$, i.e., if $J_{\mathbf{R}}(F)$ is $\mathbf{D}$-linear at each point.

In order for a function $F$ between open subsets of $\mathbf{D}^{n}$ to be bi-holomorphic we must require that $F$ be holomophic and that $\operatorname{det}_{\mathbf{D}} \frac{\partial F}{\partial z} \in \mathbf{D}^{*}$ be non-null, so that $\frac{\partial F}{\partial z}$ is invertible. 
The D-unitary group, denoted $U_{n}(\mathbf{D})$, can be defined, as in the complex case, in several equivalent ways. Given $A \in M_{n}(\mathbf{D})$, let $A^{*}=\bar{A}^{t}$, denote the conjugate transpose. Let $e_{1}, \ldots, e_{n}, T e_{1}, \ldots, T e_{n}$ denote the standard basis for $\mathbf{D}^{n}=\mathbf{R}^{n} \oplus \tau \mathbf{R}^{n}$. A set of vectors $\epsilon_{1}, \ldots, \epsilon_{n}$ in $\mathbf{D}^{n}$ is a spacelike $\mathbf{D}$-unitary basis for $\mathbf{D}^{n}$ if $\epsilon_{1}, \ldots, \epsilon_{n}, T \epsilon_{1}, \ldots, T \epsilon_{n}$ is a real orthonormal basis with $\left\langle\epsilon_{j}, \epsilon_{j}\right\rangle=1$ $\left\langle T \epsilon_{j}, T \epsilon_{j}\right\rangle=-1$ for all $j$. A matrix $A \in M_{n}(\mathbf{D})$ is called $\mathbf{D}$-unitary if one of the following equivalent conditions holds:

(1) $(A z, A z)=(z, z)$ for all $z \in \mathbf{D}^{n}$

(2) $A A^{*}=I$ or $A^{*} A=I$

(3) $A e_{1}, \ldots, A e_{n}$ is a space-like $\mathbf{D}$-unitary basis for $\mathbf{D}^{n}$.

When $n=1$, the unitary group $\mathrm{U}_{1}(\mathbf{D})$ is the space-like sphere $\left\{ \pm e^{\tau \theta}: \theta \in \mathbf{R}\right\}$ with two components. In general, $\mathrm{U}_{n}(\mathbf{D})$ has two components determined by $\operatorname{det}_{\mathbf{D}} A= \pm e^{\tau \theta}$. Let $\mathrm{U}_{n}^{+}(\mathbf{D})$ denote the identity component. Computing in null coordinates, first express $A \in M_{n}(\mathbf{D})$ as $A=e B+\bar{e} C$ with $B, C \in M_{n}(\mathbf{R})$, then we see that: $A \in \mathrm{U}_{n}(\mathbf{D}) \Longleftrightarrow A=e B+\bar{e}\left(B^{t}\right)^{-1}$ for some $B \in \mathrm{GL}_{n}(\mathbf{R})$, and that $A \in \mathrm{U}_{n}^{+}(\mathbf{D}) \Longleftrightarrow B \in \mathrm{GL}_{n}^{+}(\mathbf{R})$. Thus $\mathrm{U}_{n}^{+}(\mathbf{D}) \cong \mathrm{GL}_{n}^{+}(\mathbf{R})$.

The special unitary group $\mathrm{SU}_{n}(\mathbf{D})$ is defined by the additional condition: $\operatorname{det}_{\mathbf{D}} A=1$, i.e., $A=e B+\bar{e}\left(B^{t}\right)^{-1}$ with $B \in \mathrm{SL}_{n}(\mathbf{R})$. Thus $\mathrm{SU}_{n}(\mathbf{D}) \cong \mathrm{SL}_{n}(\mathbf{R})$. Note that $\left(B^{t}\right)^{-1}=(\operatorname{det} B)^{-1} \widetilde{B}$.

\section{The Special Lagrangian Calibration.}

The Special Lagrangian calibration, and its associated differential equation, which were introduced in $\left[\mathrm{HL}_{1}\right]$, have a complete analogue in $\mathbf{D}^{n}$. This work, due to Jack Mealy $\left[\mathrm{M}_{1,2}\right]$, will be presented in this and the following sections.

Some Preliminaries. A real $n$-plane $P$ in $\mathbf{D}^{n}$ is Lagrangian if $\left.\omega\right|_{P}=0$. Equivalently, $\left\langle z^{\prime}, T z\right\rangle=$ $\omega\left(z^{\prime}, z\right)=0$ for all $z, z^{\prime} \in P$, i.e., $z \in P \Rightarrow T z \in P^{\perp}$. Let LAG denote the set of all Lagrangian $n$-planes. A real $n$-plane $P$ in $\mathbf{D}^{n}$ is space-like if the inner product $\langle\cdot, \cdot\rangle$ on $\mathbf{D}^{n}$, when restricted to $P$, is positive definite. In particular, $P \cap \tau \mathbf{R}^{n}=\{0\}$ so that $P$ can be graphed over $\mathbf{R}^{n}$ in $\mathbf{D}^{n}=\mathbf{R}^{n} \oplus \tau \mathbf{R}^{n}$ by a matrix $A \in M_{n}(\mathbf{R})$. Pulling back the inner product $\langle\cdot, \cdot\rangle$ on $\mathbf{D}^{n}$ to $\mathbf{R}^{n}$ by the graphing parameterization $x \mapsto x+\tau A x$ yields $\langle x, x\rangle-\langle A x, A x\rangle$. Hence

$$
P \text { is spacelike } \quad \Longleftrightarrow \quad I-A^{t} A>0 \text {. }
$$

Let $G_{\text {space }}\left(n, \mathbf{D}^{n}\right)$ denote the Grassmannian of oriented space-like $n$-planes in $\mathbf{D}^{n}$. There are two connected components $G_{\text {space }}^{+}\left(n, \mathbf{D}^{n}\right)$ and $G_{\text {space }}^{-}\left(n, \mathbf{D}^{n}\right)$. The graph of $A$, when given the orientation from $\mathbf{R}^{n}$, is defined to be in $G_{\text {space }}^{+}\left(n, \mathbf{D}^{n}\right)$, and when given the opposite orientation, is defined to be in $G_{\text {space }}^{-}\left(n, \mathbf{D}^{n}\right)$.

Recall that an oriented $p$-plane in a real vector space $V$ does not uniquely determine a simple (or decomposable) vector $\xi \in \Lambda^{p} V$ by choosing an oriented basis $\epsilon_{1}, \ldots, \epsilon_{p}$ and defining $\xi=\epsilon_{1} \wedge \cdots \wedge$ $\epsilon_{p}$. However, $\xi$ is determined up to a positive scale. If $V$ is equipped with a positive definite inner product, then $\xi \in \Lambda^{p} V$ is uniquely determined by requiring $\epsilon_{1}, \ldots, \epsilon_{p}$ to be an oriented orthonormal basis, and the Grassmannian of oriented $p$-planes in $V$, denoted $G(p, V)$, can be identified with a subset of $\Lambda^{p} V$. This is not possible if $V$ is equipped with an inner product such as the one on $\mathbf{D}^{n}$. However, if $\xi \in G_{\text {space }}\left(n, \mathbf{D}^{n}\right)$ is an oriented space-like $n$-plane in $\mathbf{D}^{n}$, then again by requiring the basis $\epsilon_{1}, \ldots, \epsilon_{n}$ to be orthonormal with respect to the induced inner product, this subset $G_{\text {space }}\left(n, \mathbf{D}^{n}\right)$ of the full Grassmannian can (and will be) identified with a subset of $\Lambda_{\mathbf{R}}^{n} \mathbf{D}^{n}$.

Finally, denote by $\widetilde{\mathrm{LAG}}$ the Lagrangian $n$-planes in $\mathbf{D}^{n}$ which are both oriented and space-like. The component $\widetilde{\mathrm{LAG}^{+}}$of $\widetilde{\mathrm{LAG}}$ containing the standard oriented $\overrightarrow{\mathbf{R}}^{n}$ in $\mathbf{D}^{n}=\mathbf{R}^{n} \oplus \tau \mathbf{R}^{n}$ will be 
referred to as the set of positive oriented space-like Lagrangian $n$-planes. The sets $\widetilde{\mathrm{LAG}^{+}} \subset \widetilde{\mathrm{LAG}} \subset$ $G_{\text {space }}\left(n, \mathbf{D}^{n}\right)$ are all subsets of $\Lambda_{\mathbf{R}}^{n} \mathbf{D}^{n}$.

The Special Lagrangian Inequality. As before, let $d z=d z_{1} \wedge \cdots \wedge d z_{n}$ denote the standard $(n, 0)$-form on $\mathbf{D}^{n}$.

Lemma 4.1. Suppose $\xi^{\prime} \in \Lambda_{\mathbf{R}}^{n} \mathbf{D}^{n} \cong \Lambda^{n} \mathbf{R}^{2 n}$ is a real $n$-vector and $A \in M_{n}(\mathbf{D})$. Then

$$
(d z)\left(A \xi^{\prime}\right)=\left(\operatorname{det}_{\mathbf{D}} A\right)(d z)\left(\xi^{\prime}\right)
$$

Proof. Note that $(d z)\left(A \xi^{\prime}\right)=\left(A^{t}(d z)\right)\left(\xi^{\prime}\right)=\left(\operatorname{det}_{\mathbf{D}} A^{t}\right)(d z)\left(\xi^{\prime}\right)=\left(\operatorname{det}_{\mathbf{D}} A\right)(d z)\left(\xi^{\prime}\right)$.

In analogy with the complex case each $\xi \in \widetilde{\mathrm{LAG}}^{+}$has a phase $\theta \in \mathbf{R}$.

Corollary 4.2. If $\xi \in \widetilde{\mathrm{LAG}}^{+}$, then

$$
(d z)(\xi)=e^{\tau \theta} \quad \text { for some } \theta \in \mathbf{R}
$$

Proof. Since $(d z)\left(\xi_{0}\right)=1$ where $\xi_{0}=\overrightarrow{\mathbf{R}}^{n}$, if $A \in \mathrm{U}_{n}^{+}(\mathbf{D})$, then

$$
(d z)\left(A \xi_{0}\right)=\operatorname{det}_{\mathbf{D}} A
$$

By (3.4)(3), each $\xi \in \widetilde{\mathrm{LAG}}^{+}$is of the form $\xi=A \xi_{0}$ with $A \in \mathrm{U}_{n}^{+}(\mathbf{D})$.

Since $A \in \mathrm{U}_{n}^{+}(\mathbf{D})$ fixes $\overrightarrow{\mathbf{R}}^{n} \Longleftrightarrow A \in \mathrm{SO}_{n}(\mathbf{R}) \subset M_{n}(\mathbf{R})$, this proves that

$$
\widetilde{\mathrm{LAG}}^{+}=\mathrm{U}_{n}^{+}(\mathbf{D}) / \mathrm{SO}_{n}(\mathbf{R})
$$

Definition 4.3. A positively oriented space-like Lagrangian $n$-plane $\xi \in \widetilde{\mathrm{LAG}}^{+}$is said to be (split) special Lagrangian or to have phase zero if $\xi=A \xi_{0}$ for some $A \in \mathrm{SU}_{n}(\mathbf{D})$. The space of all (split) special Lagrangian $n$-planes in $\mathbf{D}^{n}$ is denoted by SLAG.

Note that

$$
\widetilde{\mathrm{SLAG}}=\mathrm{SU}_{n}(\mathbf{D}) / \mathrm{SO}_{n}(\mathbf{R}) \text {. }
$$

since $\mathrm{SO}_{n}(\mathbf{R})$ is contained in $\mathrm{SU}_{n}(\mathbf{D})$, and (4.2) holds.

THEOREM 4.4. (Mealy).

$$
(\operatorname{Re} d z)(\xi) \geq 1 \text { for all } \xi \in G_{\text {space }}^{+}\left(n, \mathbf{D}^{n}\right)
$$

with equality if and only if $\xi \in \widetilde{\mathrm{SLAG}}$.

Proof. Let $\mathbf{e}=(1,0)$ and $T \mathbf{e}=(0,1)$ denote the standard basis for $\mathbf{D} \cong \mathbf{R}^{2}$. Then the dual basis for $\left(\mathbf{R}^{2}\right)^{*}$ is $\mathbf{e}^{*}=d x$ and $(T \mathbf{e})^{*}=d y$. Employing this same notation in $\mathbf{D}^{n}$, we have

$$
d z=\left(\mathbf{e}_{1}^{*}+\tau\left(T \mathbf{e}_{1}\right)^{*}\right) \wedge \cdots \wedge\left(\mathbf{e}_{n}^{*}+\tau\left(T \mathbf{e}_{n}\right)^{*}\right) .
$$

Expanding out this product expresses $d z$ as a $2^{n}$-fold sum. Each term $\alpha$ in the sum is the product of $n$ of the $2 n$ axis covectors $\mathbf{e}_{1}^{*}, \tau\left(T \mathbf{e}_{1}\right)^{*}, \ldots, \mathbf{e}_{n}^{*}, \tau\left(T \mathbf{e}_{n}\right)^{*}$. However, for any $j=1, \ldots, n$,

$$
\text { both } \mathbf{e}_{j}^{*} \text { and } \tau\left(T \mathbf{e}_{j}\right)^{*} \text { cannot be factors of } \alpha \text {. }
$$


By Proposition A.3 each $\xi \in G_{\text {space }}^{+}\left(n, \mathbf{D}^{n}\right)$ is unitarily equivalent to

$$
\xi^{\prime}=\mathbf{e}_{1} \wedge\left(\cosh \theta_{1} \mathbf{e}_{2}^{*}+\sinh \theta_{1}\left(T \mathbf{e}_{1}\right)^{*}\right) \wedge \mathbf{e}_{3} \wedge\left(\cosh \theta_{2} \mathbf{e}_{4}^{*}+\sinh \theta_{2}\left(T \mathbf{e}_{3}\right)^{*}\right) \wedge \cdots,
$$

that is, $\xi=A \xi^{\prime}$ with $A \in \mathrm{U}_{n}^{+}(\mathbf{D})$. By (4.4), (4.5) and (4.6) we have

$$
(d z)\left(\xi^{\prime}\right)=\cosh \theta_{1} \cdots \cosh \theta_{\left[\frac{n}{2}\right]} .
$$

Hence by Lemma 4.1

$$
(d z)(\xi)=e^{\tau \theta} \cosh \theta_{1} \cdots \cosh \theta_{\left[\frac{n}{2}\right]}
$$

where $e^{\tau \theta}=\operatorname{det}_{\mathbf{D}} A$. In particular

$$
(\operatorname{Re} d z)(\xi)=\cosh \theta \cosh \theta_{1} \cdots \cosh \theta_{\left[\frac{n}{2}\right]} .
$$

Hence $(\operatorname{Re})(\xi) \geq 1$ and $=1$ if and only if all the angles are zero, i.e., $\xi^{\prime}=\mathbf{e}_{1} \wedge \cdots \wedge \mathbf{e}_{n}=\xi_{0}$, $\xi=A \xi^{\prime}=A \xi_{0}$, and $\operatorname{det}_{\mathbf{D}} A=1$.

Corollary 4.5. An oriented real $n$-plane $\xi$ in $\mathbf{D}^{n}$ is (split) special Lagrangian if and only if $\xi \in \widetilde{\mathrm{LAG}}^{+}$and $\left.\operatorname{Im} d z\right|_{\xi}=0$.

The Null Viewpoint. We now revisit the material above in null coordinates. Several points are worth mentioning. Note first that (4.3) naturally becomes

$$
\widetilde{\mathrm{SLAG}}=\mathrm{SL}_{n}(\mathbf{R}) / \mathrm{SO}_{n}(\mathbf{R})
$$

since $A \in \mathrm{SU}_{n}(\mathbf{D})$ if and only if $A=e B+\bar{e}\left(B^{t}\right)^{-1}=e B+\bar{e} \widetilde{B}$ for some $B \in \mathrm{SL}_{n}(\mathbf{R})$.

Note also that

$$
\begin{gathered}
d z=\frac{1}{2}(d u+d v)+\frac{\tau}{2}(d v-d u), \quad \text { or } \\
\operatorname{Re} d z=\frac{1}{2}(d u+d v) \quad \text { and } \quad \operatorname{Im} d z=\frac{1}{2}(d v-d u) .
\end{gathered}
$$

To prove this note that $d z_{j}=e d u_{j}+\bar{e} d v_{j}$ implies that $d z=e d u+\bar{e} d v$.

Now Corollary 4.5 has the null form:

Corollary 4.5'. An oriented real $n$-plane $\xi$ in $\mathbf{D}^{n}$ is (split) special Lagrangian if and only if $\xi \in \widetilde{\mathrm{LAG}}+$ and $\left.d u\right|_{\xi}=\left.d v\right|_{\xi}$.

\section{Split SLAG Submanifolds.}

The algebraic calculations of the previous sections apply to submanifolds. In what follows all submanifolds are assumed to have dimension $n$.

Definition 5.1. A closed oriented $C^{1}$-submanifold $M$ of an an open subset of $\mathbf{D}^{n}$ is a split SLAG (or STAG) submanifold if the oriented tangent space $\vec{T}_{z} M \in \widetilde{\mathrm{SLAG}}$ for all $z \in M$.

THEOREM 5.2. A closed oriented $C^{1}$-submanifold $M$ of an an open subset of $\mathbf{D}^{n}$ is split SLAG if and only if

(1) $M$ is Lagrangian, i.e., $\left.\omega\right|_{M}=0$,

(2) $\left.\psi\right|_{M}=0$ where $\psi \equiv \operatorname{Im} d z=\frac{1}{2}(d v-d u)$, and 
(3) $M$ is positive space-like.

Proof. Apply Corollary 4.5 and (4.10).

If $M$ satisfies only conditions (1) and (2), it will be called an unconstrained (or not necessarily space-like) split SLAG submanifold.

Each oriented space-like submanifold $N$ inherits a volume form $\operatorname{vol}_{N}$ from $\mathbf{D}^{n}$.

THEOREM 5.3. Suppose that $(M, \partial M)$ is a compact oriented submanifold with boundary in $\mathbf{D}^{n}$. If $M$ is split SLAG, then $M$ is volume-maximizing, i.e.,

$$
\operatorname{vol}(M) \geq \operatorname{vol}(N)
$$

for any other positive space-like compact submanifold $N$ with $\partial N=\partial M$.

Equality holds in (5.1) if and only if $N$ is also split SLAG.

Proof. We have

$$
\operatorname{vol}(M)=\int_{M} \operatorname{Re} d z=\int_{N} \operatorname{Re} d z \geq \operatorname{vol}(N)
$$

by Theorem 4.4, Stokes' Theorem, and Theorem 4.4 again. Equality in (5.1) implies Re $\left.d z\right|_{N} \equiv$ $\operatorname{vol}(N)$ and therefore $N$ is split SLAG by Theorem 4.4 once again.

Remark 5.4. (Split $\mathbf{S L A G} \mathbf{A G}_{\theta}$-Calibrations). As in the complex case, each rotation of the calibration $\operatorname{Re} d z$ above gives a new calibration $\operatorname{Re}\left(e^{-\tau \theta} d z\right)$ on positively oriented space-like submanifolds. The corresponding calibrated submanifolds are the Lagrangian submanifolds with the property that the restriction

$$
\left.\operatorname{Im}\left(e^{-\tau \theta} d z\right)\right|_{M}=0
$$

or equivalently, that $\left.\operatorname{Re}\left(e^{-\tau \theta} d z\right)\right)\left.\right|_{M}=d \operatorname{vol}_{M}$.

Note that Corollary 4.2 provides a fibration

$$
\widetilde{\mathrm{LAG}}^{+} \longrightarrow \mathbf{R}
$$

whose fibre at $\theta \in \mathbf{R}$ is exactly the set $\widetilde{\mathrm{SLAG}_{\theta}}$ of $n$-planes in $G_{\text {space }}^{+}\left(n, \mathbf{D}^{n}\right)$ calibrated by $\operatorname{Re}\left(e^{-\tau \theta} d z\right)$.

We shall not discuss these other cases since they are completely equivalent to the case $\theta=0$. If $A \in \mathrm{U}_{n}^{+}(\mathbf{D})$ with $\operatorname{det}_{\mathbf{D}}(A)=e^{\tau \theta}$, then this isometry $A: \mathbf{D}^{n} \rightarrow \mathbf{D}^{n}$ carries SLAG-submanifolds

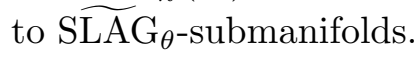

\section{Split SLAG Graphs in $\mathbf{D}^{n}$.}

Locally the graph of a smooth map $F: \mathbf{R}^{n} \rightarrow \mathbf{R}^{n}$ is Lagrangian in $\mathbf{R}^{2 n}$ if and only if $F=d f$ for some potential function $f$. This is because $\omega=\sum_{j} d x_{j} \wedge d y_{j}$ vanishes when restricted to $M$ if and only if $\left.\sum_{j} y_{j} d x_{j}\right|_{M}$ is $d$-closed. This is equivalent to requiring the Jacobian of $F$ to be symmetric, in which case it equals the hessian of the potential $f$.

Any space-like submanifold $M$ is locally the graph of a smooth map from $\mathbf{R}^{n}$ to $\tau \mathbf{R}^{n}$ since $T_{z} M \cap \tau \mathbf{R}^{n}=\{0\}$ for all $z \in M$. Furthermore, in Proposition 7.6 below we show that if $M$ is a closed space-like Lagrangian submanifold of a tube $\Omega \times \tau \mathbf{R}^{n} \subset \mathbf{R}^{n} \times \tau \mathbf{R}^{n}=\mathbf{D}^{n}$, then the projection $\left.\pi\right|_{M}: M \rightarrow \Omega$ is a covering map. In particular, if $\Omega$ is simply-connected, then each connected component of $M$ is the graph of a gradient $d f$ for some potential function $f: \Omega \rightarrow \mathbf{R}$. This makes the following theorem particularly relevant. 
THEOREM 6.1. (Mealy). Suppose that $f$ is a smooth real-valued function defined on an open subset of $\mathbf{R}^{n}$. Let $M$ denote the oriented graph of the gradient $F=d f$ in $\mathbf{D}^{n}=\mathbf{R}^{n} \oplus \tau \mathbf{R}^{n}$. Then $M$ is split special Lagrangian $\Longleftrightarrow M$ is space-like and

$$
\sum_{k=0}^{[(n-1) / 2]} \sigma_{2 k+1}(\operatorname{Hess} f)=\operatorname{Im} \operatorname{det}_{\mathbf{D}}(I+\tau \operatorname{Hess} f)=0 \text {. }
$$

Furthermore $M$ is space-like $\Longleftrightarrow$

$$
-I<\operatorname{Hess} f<I .
$$

or, equivalently, the graphing map of $d f$ is uniformly Lipschitz with Lipschitz constant 1.

Proof. The tangent plane $P$ to $M$ at a point is the graph of $A \in M_{n}(\mathbf{R})$ in $\mathbf{R}^{n} \oplus \tau \mathbf{R}^{n}$, and is parameterized by $x \mapsto x+\tau A x, x \in \mathbf{R}^{n}$. Pulling back the symplectic form $\omega$ from $P$ to $\mathbf{R}^{n}$ gives $\omega\left((x, A x),\left(x^{\prime}, A x^{\prime}\right)\right)=\left\langle x, A x^{\prime}\right\rangle-\left\langle A x, x^{\prime}\right\rangle$ so that, as noted above, $P$ is Lagrangian $\Longleftrightarrow A$ is symmetric. Pulling back the quadratic form $\langle\cdot, \cdot\rangle$ yields $\langle x, x\rangle-\langle A x, A x\rangle$. Hence, as noted in (4.0),

$$
P \equiv \operatorname{graph} A \text { is spacelike } \Longleftrightarrow A^{t} A<I \text {. }
$$

It suffices to prove Theorem 6.1 when $M$ is a plane. This case is handled explicitly in the following.

Proposition 6.2. Suppose $\xi=\operatorname{graph} A$ is the oriented graph in $\mathbf{D}^{n}=\mathbf{R}^{n} \oplus \tau \mathbf{R}^{n}$ of a matrix $A \in M_{n}(\mathbf{R})$. Then $\xi \in \widehat{\mathrm{SLAG}} \Longleftrightarrow A$ is symmetric with $-I<A<I$ and

$$
\operatorname{Im} \operatorname{det}_{\mathbf{D}}(I+\tau A)=0 .
$$

Proof. We have already shown that $\xi$ is space-like and Lagrangian (i.e., $A \in \widetilde{\mathrm{LAG}}$ ) $\Longleftrightarrow A$ is symmetric and $A^{2}<I$, (which is equivalent to $-I<A<I$ for symmetric $A$ ). Since the space of such oriented graphs is connected and contains $\xi_{0}=\overrightarrow{\mathbf{R}}^{n}$ (take $A=0$ ), this proves that

$$
\xi=\operatorname{graph} A \in \widetilde{\mathrm{LAG}}^{+} \Longleftrightarrow A \text { is symmetric and }-I<A<I \text {. }
$$

Now the pull-back of $d z$ under the D-linear map $I+\tau A: \mathbf{D}^{n} \rightarrow \mathbf{D}^{n}$ is equal to $\operatorname{det}_{\mathbf{D}}(I+\tau A) d z$. Hence, the pull-back of $\left.d z\right|_{\xi}$ to $\mathbf{R}^{n}$ under the graphing map $x \mapsto x+\tau A x$ equals $\operatorname{det}_{\mathbf{D}}(I+\tau A) d x$. Hence (6.3) holds $\left.\Longleftrightarrow \operatorname{Im} d z\right|_{\xi}=0$.

Corollary 4.5 completes the proof of Proposition 6.2 and Theorem 6.1.

The Null Viewpoint. It is frequently more interesting to consider graphs over the null $n$-plane $e \mathbf{R}^{n}$ in $\mathbf{D}^{n}=e \mathbf{R}^{n} \oplus \bar{e} \mathbf{R}^{n}$.

Each space-like submanifold $M$ in $\mathbf{D}^{n}$ is locally the graph of a smooth map from a domain $\Omega \subseteq \mathbf{R}^{n}$ to $\bar{e} \mathbf{R}^{n}$ since $T_{z} M \cap \bar{e}=\{0\}$ for all $z \in M$. Furthermore, since $\omega=\sum_{j} d u_{j} \wedge d v_{j}$ we see that if $M$ is also Lagrangian and $\Omega$ is simply-connected, then this graph is the gradient $d f$ of a potential function $f$ on $\Omega$.

THEOREM 6.3. Suppose that $f$ is a smooth function defined on an open subset of $\mathbf{R}^{n}$. Let $M$ denote the oriented graph of the gradient $F=d f$ in $e \mathbf{R}^{n} \oplus \bar{e} \mathbf{R}^{n} \equiv \mathbf{D}^{n}$. Then

$$
M \text { is split special Lagrangian } \Longleftrightarrow f \text { is convex and } \operatorname{det}_{\mathbf{R}} \operatorname{Hess} f \equiv 1 \text {. }
$$


Proof. The standard plane $\xi_{0}=\overrightarrow{\mathbf{R}}^{n}$ is the graph of $A=I \in M_{n}(\mathbf{R})$. For any $A \in M_{n}(\mathbf{R})$, the previous discussion shows that $\xi=\operatorname{graph} A$ is Lagrangian $\Longleftrightarrow A$ is symmetric. The quadratic form $\langle\cdot, \cdot\rangle$ restricted to the graph of $A$ pulls back to $\langle e u+\bar{e} A u, e u+\bar{e} A u\rangle=2\langle e u, \bar{e} A u\rangle=\langle u, A u\rangle$ since $e u$ and $\bar{e} A u$ are null and $\langle e, \bar{e}\rangle=\frac{1}{2}$ in $\mathbf{D}$. Thus:

$$
\operatorname{graph} A \text { is spacelike } \Longleftrightarrow A+A^{t}>0 \text {. }
$$

By a connectivity argument it follows that: for the oriented graph $\xi$ of $A \in \mathbf{M}_{n}(\mathbf{R})$

$$
\xi=\operatorname{graph} A \in \widetilde{\mathrm{LAG}}^{+} \Longleftrightarrow A \text { is symmetric and } A>0 \text {. }
$$

Proposition 6.4. One has $\xi \in \widetilde{\mathrm{SLAG}} \Longleftrightarrow \quad \xi$ is the oriented graph over $e \mathbf{R}^{n}$ in $\mathbf{D}^{n}=$ $e \mathbf{R}^{n} \oplus \bar{e} \mathbf{R}^{n}$ of a positive definite symmetric matrix $A \in M_{n}(\mathbf{R})$ satisfying

$$
\operatorname{det}_{\mathbf{R}} A=1 \text {. }
$$

Proof. As noted above, each space-like $n$-plane $P$ can be graphed over $e \mathbf{R}^{n}$ since $P \cap e \mathbf{R}^{n}=\{0\}$. Under the graphing map $u \mapsto e u+\bar{e} A u$, the $n$-form $d u$ pulls back to $d u$ and the $n$-form $d v$ pulls back to $\left(\operatorname{det}_{\mathbf{R}} A\right) d u$. Thus by Corollary $4.5^{\prime}$ we have $\xi=\operatorname{graph} A \in \widehat{\mathrm{SLAG}} \Longleftrightarrow \xi \in \widetilde{\mathrm{LAG}}^{+}$and $\operatorname{det} A=1$. This completes the proof of both Proposition 6.4 and Theorem 6.3.

\section{Removable Singularities for split SLAG varieties.}

In this section we show that split SLAG subvarieties tend to be less singular than their elliptic cousins. For example, any connected split SLAG submanifold in a simply-connected tube domain $\Omega \times \tau \mathbf{R}^{n}$ is the graph of a mapping with potential function satisfying (6.1). Moreover, singularities in codimension $>2$ tend to be removable. In particular, there are no irreducible split SLAG cones with isolated singularity in $\mathbf{D}^{n}$ for $n \geq 3$.

The proofs of these results, which follow, illustrate an interesting interplay between the $z$ coordinate and the null-coordinate viewpoints. This shift of coordinates, sometimes called the Cayley transformation, turns out to be quite useful in the analysis.

The first main theorem of this section is the following.

THEOREM 7.1. (Removable Singularities I). Let $\Omega \subset \mathbf{R}^{n}$ be a convex domain in $\mathbf{R}^{n}$ and consider the "tube" domain $\Omega \times \tau \mathbf{R}^{n} \subset \mathbf{R}^{n} \oplus \tau \mathbf{R}^{n}=\mathbf{D}^{n}$. Let $\Sigma \subset \Omega$ be a compact subset of Hausdorff $(n-2)$-measure zero. Then any closed, connected split SLAG submanifold

$$
M \subset(\Omega-\Sigma) \times \tau \mathbf{R}^{n}
$$

has closure in $\Omega \times \tau \mathbf{R}^{n}$ which is the graph of a real analytic mapping $F: \Omega \rightarrow \mathbf{R}^{n}$. Furthermore, $F=\nabla f$ where $f: \Omega \rightarrow \mathbf{R}$ satisfies the equation (6.1).

Corollary 7.2. (Absence of Cones). Suppose $C \subset \mathbf{D}^{n}$ is a split SLAG cone which is regular and connected outside the origin. Then $C$ is a SLAG $n$-plane.

Proof of Corollary 7.2. The proof is immediate for $n \geq 3$ - one takes $\Omega=\mathbf{R}^{n}$ and $\Sigma=\{0\}$. The case $n=2$ follows from Theorem F.3 in Appendix F and the fact that complex cones of complex dimension 1 are planes. 
Remark 7.3. This is in sharp contrast to the non-split case where SLAG-cones exist in all dimensions and there are many SLAG-varieties with singularities of high codimension. (See $\left[\mathrm{HL}_{1}\right]$, $\left[\mathrm{J}_{*}\right],\left[\mathrm{H}_{*}\right],\left[\mathrm{HK}_{*}\right]$ and the references therein). Also in coassociative geometry there is a coassociative cone, smooth outside 0 in $\mathbf{R}^{7}$ which is the graph of a Lipschitz map [ $\left.\mathrm{HL}_{1}\right]$.

Remark 7.4. The codimension-2 hypothesis in Theorem 7.1 is close to best possible. There do exist split SLAG subvarieties in all dimensions $n \geq 2$ with codimension- 2 singularities. When $n=2$ they appear because split SLAG subvarieties are complex curves for a certain complex structure (see Appendix E). The higher dimensional cases are just products of these with $\mathbf{R}^{n-2}$.

On the other hand we still do not know of any non-trivial (irreducible) split SLAG cones.

Corollary 7.5. (Removable Singularities II). Let $\Omega \subset \mathbf{R}^{n}$ be a domain in $\mathbf{R}^{n}$ and suppose $\Sigma \subset \Omega$ is any closed subset of linear-measure zero. Then any closed, split SLAG submanifold $M \subset(\Omega-\Sigma) \times \tau \mathbf{R}^{n}$ has closure in $\Omega \times \tau \mathbf{R}^{n}$ which is an immersed real analytic split SLAG submanifold of $\Omega \times \mathbf{R}^{n}$.

Proof of Corollary 7.5. Fix $(x, y) \in \bar{M}$. Choose a ball $B=B_{r}(x) \subset \Omega$ of radius $r$ such that $\partial B \cap \Sigma=\emptyset$. One now applies Theorem 7.1 to the tube domain $B \times \mathbf{R}^{n}$ to conclude that the closure of each connected component of $M \cap(B-\Sigma) \times \mathbf{R}^{n}$ is the graph of an analytic mapping $B \rightarrow \mathbf{R}^{n}$.

Proof of Theorem 7.1. We begin with the following observation.

Proposition 7.6. Let $\Omega \subset \mathbf{R}^{n}$ be a domain in $\mathbf{R}^{n}$ and consider the "tube" domain $T \equiv \Omega \times \tau \mathbf{R}^{n} \subset$ $\mathbf{R}^{n} \oplus \mathbf{R}^{n}=\mathbf{D}^{n}$ with projection $\pi: T \rightarrow \Omega$. If $M \subset T$ is a closed (embedded) submanifold which is space-like and Lagrangian, then $\left.\pi\right|_{M}: M \rightarrow \Omega$ is a covering map.

If, in addition, $\Omega$ is simply-connected, then each component of $M$ is the graph of $\nabla f$ for some potential function $f: \Omega \rightarrow \mathbf{R}$, and this graph mapping $F=\nabla f$ is 1-Lipschitz.

Proof. To begin we note that from (6.2) we have the following:

$$
\left\|\pi_{*}(v)\right\| \geq \frac{1}{\sqrt{2}}\|v\| \text { for all } v \in T(M)
$$

Now fix a point $x \in \Omega$ such that $x=\pi(y)$ for some $y \in M$. Let $\gamma:[0,1] \rightarrow \Omega$ be a smooth curve with $\gamma(0)=x$. From 7.1 one easily concludes that there exists a lift $\widetilde{\gamma}:[0,1] \rightarrow M$ with $\widetilde{\gamma}(0)=y$ and $\pi \circ \widetilde{\gamma}=\gamma$. (The set of $t \in[0,1]$ such that $\left.\gamma\right|_{[0, t]}$ can be lifted, is both open and closed.)

Fix a closed ball $B=B(x, r)$ about $x$ which is contained in $\Omega$. Then lifting radial lines from $x$ (as above) gives a mapping $\varphi: B \rightarrow M$ with $\varphi(x)=y$ and $\pi \circ \varphi=\operatorname{Id}_{B}$. It now follows that for each connected component $M_{0}$ of $M$, the mapping $\pi_{0}=\left.\pi\right|_{M_{0}}: M_{0} \rightarrow \Omega$ is a covering map, i.e., $\pi_{0}$ is surjective and every point $x$ has a neighborhood $B$ which is evenly covered by $\pi_{0}$. The first assertion is proved.

If $\Omega$ is simply-connected and $M$ connected, then by elementary covering space theory $\pi=$ $\left.\pi\right|_{M}: M \rightarrow \Omega$ is a diffeomorphism. Hence $M$ is the graph of a function $F: \Omega \rightarrow \mathbf{R}^{n}$, which, since $M$ is Lagrangian and $\Omega$ is simply-connected, is the gradient $F=\nabla f$ of a scalar function $f: \Omega \rightarrow \mathbf{R}$.

Since $M$ is also space-like, we have from (6.2) that

$$
-I<\operatorname{Jac}(F)<I \quad \text { on } \Omega
$$

Hence $F$ is 1-Lipschitz and the proof is complete. 
Returning to Theorem 7.1, we apply Proposition 7.6 to the simply-connected domain $\Omega-\Sigma$ to conclude that $M$ is the graph of a gradient $F=\nabla f: \Omega-\Sigma \rightarrow \mathbf{R}^{n}$ which satisfies (7.2). The map $F$ extends uniquely to a 1-Lipschitz mapping $\hat{F}: \Omega \rightarrow \mathbf{R}^{n}$ which can be written as the gradient $\hat{F}=\nabla \hat{f}$ of a unique extension of $f$ to $\Omega$. It follows that the closure of $M$ in $\Omega$ is

$$
\bar{M}=M \cup \Sigma_{M}
$$

where

$$
\Sigma_{M} \equiv(I \times \hat{F})(\Sigma) \text { is compact with Hausdorff }(n-2) \text { measure zero. }
$$

We now pass to null coordinates $(u, v)$ on $\mathbf{D}^{n}$ and consider the projection of $\bar{M}$ onto the $u$-axis. Composing this projection with the graphing map $F$ gives a mapping $G=F+I: \Omega \rightarrow \mathbf{R}^{n}$ (i.e., $G(x)=F(x)+x$ for $x \in \Omega$ ). Note that $G=\nabla g$ where $g(x)=f(x)+\frac{1}{2}|x|^{2}$. From (7.2) we see that this map satisfies

$$
0<\operatorname{Jac} G<2 I \quad \text { on } \Omega-\Sigma \text {. }
$$

In particular, the potential $g$ is strictly convex on $\Omega-\Sigma$. It follows that for any two points $x, y \in \Omega$, we have that

$$
G(x) \neq G(y) \text { unless the segment } \overline{x y} \subset \Sigma .
$$

In particular, $G$ is injective on $\Omega-\Sigma$. Thus $\left.G\right|_{\Omega-\Sigma}$ is a diffeomorphism onto its image. Moreover we have the following.

Lemma 7.7. The map $\left.G\right|_{\Omega-\Sigma}: \Omega-\Sigma \rightarrow \Omega^{\prime}-\Sigma^{\prime}$ is a diffeomorphism, where $\Omega^{\prime} \equiv G(\Omega)$ and $\Sigma^{\prime} \equiv G(\Sigma) \subset \subset \Omega^{\prime}$.

Proof. By (7.4) and the strict convexity we know that if $x \in \Omega-\Sigma$ and $y \in \Sigma$, then $G(x) \neq G(y)$. Hence, $G(\Omega-\Sigma) \cap G(\Sigma)=\emptyset$. By shaving $\Omega$ slightly we may assume it has a smooth boundary $\partial \Omega$ which does not meet $\Sigma$ and along which $G$ is a diffeomorphism. Evidently $G(\Sigma)$ will be a compact subset of the bounded component of $\mathbf{R}^{n}-G(\partial \Omega)$.

Since $G$ is 2-Lipschitz, the Hausdorff 2-measure $\mathcal{H}\left(\Sigma^{\prime}\right)=0$. It follows that $\Omega^{\prime}-\Sigma^{\prime}$ is simplyconnected. Now note that $\bar{M}$ is the graph in null coordinates of a mapping $v=H(u)$ where $H: \Omega^{\prime} \rightarrow \mathbf{R}^{n}$ is well defined and smooth except possibly over points of $\Sigma^{\prime}$. (Points where it is not well defined correspond to points lying on non-trivial straight line segments in $\Sigma$ ). Hence, in $\Omega^{\prime}-\Sigma^{\prime}$ we know that $H=\nabla h$ where $h$ is a smooth strictly convex function which satisfies the equation

$$
\operatorname{det} \operatorname{Hess}(h)=1
$$

on $\Omega^{\prime}-\Sigma^{\prime}$. Here we are using the assumption that $M$ is SLAG and Theorem 6.3.

Now any convex function defined on $\Omega^{\prime}-S$ where $S \subset \Omega$ is closed with $\mathcal{H}^{n-2}(S)=0$, extends as a convex function across $S$. Thus $h$ is well defined and convex on $\Omega^{\prime}$

We claim that $h$ is a weak solution of equation (7.5) (and therefore a viscosity solution by $\left[\mathrm{C}_{3}\right.$, Lemma 3]) on all of $\Omega^{\prime}$. To see this note that for any Borel set $\mathcal{O} \subset \Omega^{\prime}-\Sigma^{\prime}$ equation (7.5) implies that

$$
\operatorname{meas}(\nabla h(\mathcal{O}))=\operatorname{meas}(\mathcal{O}) .
$$

However, by (7.3) this holds also for any Borel set $\mathcal{O} \subset \Omega^{\prime}$.

We now apply deep results of Caffarelli (Theorem 7.8 below) to conclude that $h$ is real analytic in $\Omega^{\prime}$. Hence, $M=\operatorname{graph}(\nabla h)$ is a real analytic submanifold of $\Omega \times \mathbf{R}^{n}$. Thus the graph of $F=\nabla f$ over $\Omega$ is real analytic. Since $F$ is 1 -Lipschitz, it is also real analytic on $\Omega$.

The following is well-known but not explicitly stated in the literature. 
THEOREM 7.8. (Caffarelli $\left[\mathbf{C}_{2,3}\right]$ ). Let $h$ be a viscosity solution of (7.5) on a bounded domain $\Omega \subset \mathbf{R}^{n}$ with smooth boundary $\partial \Omega$. Assume $h$ is smooth in a neighborhood of $\partial \Omega$ in $\bar{\Omega}$. Then $h$ is real analytic in $\Omega$.

Example 7.9. Take the union of the standard $\mathbf{R}^{n} \subset \mathbf{D}^{n}$ with the $n$-plane: $y_{1}=\cdots=y_{n-2}=$ $0, y_{n-1}=\lambda x_{n-1}, y_{n}=-\lambda x_{n}$. Both are split SLAG. This union is singular (but not irreducible) along the intersection $\left(\mathbf{R}^{n-2} \times\{0\}\right) \times\{0\}$.

Example 7.10. The following classical example of Pogorelov is of interest in this context. Let $(u, v)$ be null coordinates on $\mathbf{D}^{n}$, and write $u=\left(u_{1}, \mathbf{u}\right)$ and $v=\left(v_{1}, \mathbf{v}\right)$ for $\mathbf{R}^{n}=\mathbf{R} \times \mathbf{R}^{n-1}$. Consider the potential

$$
\varphi\left(u_{1}, \mathbf{v}\right)=-k|\mathbf{v}|^{\frac{2(n-1)}{n-2}} f\left(u_{1}\right)
$$

where $f(t)$ satisfies $f^{\prime \prime}+f=0$ and $f>0$. The graph $M$ of the gradient of $\varphi$ is:

$$
\begin{aligned}
v_{1} & =-k|\mathbf{v}|^{\frac{2(n-1)}{n-2}} f^{\prime}\left(u_{1}\right) \\
\mathbf{u} & =-k \frac{2(n-1)}{n-2}|\mathbf{v}|^{\frac{2}{n-2}} f\left(u_{1}\right) \mathbf{v}
\end{aligned}
$$

Note that $M$ is real analytic if $n=3$, but just $C^{1, \alpha}$ along the $u_{1}$-axis if $n \geq 4$ (where $\alpha=\left(\left[\frac{n}{2}\right]-\frac{1}{2}\right)^{-1}$ when $n$ is odd, and $\alpha=\left(\frac{n}{2}-1\right)^{-1}$ when $n$ is even). The tangent plane at points on the $u_{1}$-axis is the $\left(u_{1}, \mathbf{v}\right)$-plane, so $M$ is being graphed over its tangent plane at these points.

With the right choice of $k, M$ is an example of a split SLAG manifold outside of a singular line which is null. In particular, when $n=3, M$ is a real analytic unconstrained split SLAG submanifold. Outside the $u_{1}=x_{1}-y_{1}$ axis it is split SLAG, but at points on this axis it is not split SLAG since this axis is tangent to $M$ and null.

Remark 7.10. Although the discussion in this section was carried out without defining the notion of a (singular) split SLAG variety, we call Appendix D to the reader's attention for this definition.

\section{Double Manifolds (D-Manifolds).}

Certain standard notions from complex analysis can now be carried over to $\mathbf{D}$-analysis.

Definition 8.1. A double manifold or D-manifold is a smooth manifold $X$ equipped with an atlas of charts $\left\{\left(U_{\alpha}, \psi_{\alpha}\right)\right\}_{\alpha}, \psi_{\alpha}: U_{\alpha} \rightarrow \mathbf{D}^{n}$, whose transition functions are $\mathbf{D}$-holomorphic and orientation-preserving, that is, the transition functions are of the form

$$
z^{\prime}=F(z) \quad \text { with } \frac{\partial F}{\partial \bar{z}}=0 \quad \text { and } \quad \operatorname{det}_{\mathbf{D}}\left(\frac{\partial F}{\partial z}\right) \in \mathbf{D}^{+}
$$

or equivalently

$$
u^{\prime}=f(u), \quad v^{\prime}=g(v) \quad \text { with } f, g \text { orientation preserving. }
$$

Let's examine these manifolds from the two points of view. In the complex picture, we have a Dolbeault decomposition

$$
\left(\Lambda^{k} T^{*} X\right) \otimes_{\mathbf{R}} \mathbf{D}=\bigoplus_{p+q=k} \Lambda^{p, q}(X)
$$


where, in local holomorphic coordinates $\left(z_{1}, \ldots, z_{n}\right), \Lambda^{p, q}$ is spanned by forms of the type $d z_{i_{1}} \wedge$ $\cdots \wedge d z_{i_{p}} \wedge d \bar{z}_{j_{1}} \wedge \cdots \wedge d \bar{z}_{j_{q}}$. Let $\mathcal{E}^{p, q}(X)$ denote the smooth sectons of $\Lambda^{p, q}(X)$. Then the operators

$$
\partial: \mathcal{E}^{p, q}(X) \longrightarrow \mathcal{E}^{p+1, q}(X) \quad \text { and } \quad \bar{\partial}: \mathcal{E}^{p, q}(X) \longrightarrow \mathcal{E}^{p, q+1}(X)
$$

already given in local holomorphic coordinates by (3.1), are well defined on the manifold $X$. A D-valued function $F$ defined on an open subset of $X$ is holomorphic if and only if $\bar{\partial} F=0$.

Suppose now that we rewrite our local charts on $X$ in terms of null coordinates $(u, v)$ in $\mathbf{D}^{n}=e \cdot \mathbf{R}^{n} \oplus \bar{e} \cdot \mathbf{R}^{n}$. Then from (8.2) it is clear that $X$ is furnished with two transversal $n$-dimensional oriented foliations $\mathcal{F}^{+}$and $\mathcal{F}^{-}$given locally by the $u$ and $v$ coordinate planes respectively. Taking the tangent plane fields $N^{ \pm} \equiv \mathcal{F}^{ \pm}$to be the \pm 1 -eigenspaces defines the structure endomorphism

$$
\mathbf{T}: T(X) \rightarrow T(X) \quad \text { with } \mathbf{T}^{2}=I d .
$$

which represents scalar multiplication by $\tau$ on the tangent spaces (cf. (2.3)). (This is the analogue of $J: T(X) \rightarrow T(X)$ with $J^{2}=-I d$ in the complex case.) Indeed from this point of view a D-manifold is simply a pair $(X, \mathbf{T})$, where $\mathbf{T}^{2}=I d$ with eigenbundles $N^{ \pm}$of equal dimension both of which are integrable. (If one drops the integrability condition, this becomes an almost $\mathbf{D}$ manifold.) Even more simply, a $\mathbf{D}$-manifold is a $2 n$-dimensional manifold with a pair of transversal $n$-dimensional oriented foliations. When $n=1$ this is equivalent to a (fully oriented) conformal Lorentzian structure on the surface.

Remark 8.2. Any product $X=M_{1} \times M_{2}$ of $n$ manifolds is certainly a $\mathbf{D}$-manifold (with the foliations given by the factors). However, D-manifolds can be much more complicated. Let $\mathcal{S}_{k}$ be a 2 -dimension foliation on a compact 3-manifold $M_{k}, k=1,2$, and let $\mathcal{L}_{k}$ be a 1-dimensional foliation determined by a line field transversal to $\mathcal{S}_{k}$. Then define the transversal 3-dimensional foliations $\mathcal{F}_{1} \equiv \mathcal{S}_{1} \times \mathcal{L}_{2}$ and $\mathcal{F}_{2} \equiv \mathcal{L}_{1} \times \mathcal{S}_{2}$ on $M_{1} \times M_{2}$. By a theorem of J. Wood [Wo] any 2-plane field on a 3-manifold is homotopic to a integrable one. Any such foliation can then be modified by introducing many Reeb components. The 1-dimensional foliations can often be constructed to have dense orbits.

Remark 8.3. (D-submanifolds). A D-submanifold is a submanifold whose tangent spaces are T-invariant with \pm 1-eigenspaces of the same dimension. This second condition is not automatic, but if it holds at a point, it holds on the connected component of that point. These subspaces are automatically integrable, with leaves given by intersection with the leaves of the ambient manifold.

The graphs of a $\mathbf{D}$-holomorphic mapping $f: X \rightarrow Y$ is a $\mathbf{D}$-submanifold. So also is $f^{-1}(p)$ if $p$ is a regular value of $f$.

We now address the question of $\mathbf{D}$-line bundles and $\mathbf{D}$-vector bundles. A $\mathbf{D}$-line bundle on an arbitrary manifold is a family of free $\mathbf{D}$-modules over $X$ which is locally isomorphic to $U \times \mathbf{D}$ with transition functions which are smooth $\mathbf{D}^{+}$-valued functions.

Definition 8.4. A holomorphic line bundle on a $\mathbf{D}$-manifold is a $\mathbf{D}$ line bundle whose transition functions can be chosen to be $\mathbf{D}$-holomorphic.

Let $\mathcal{D}$ denote the sheaf of germs of $\mathbf{D}$-holomorphic functions on a $\mathbf{D}$-manifold $X$, and let $\mathcal{D}^{+}$ denote the sheaf of germs of holomorphic $\mathbf{D}^{+}$-valued functions on $X$. Then the Čech cohomology group

$$
H^{1}\left(X, \mathcal{D}^{+}\right)
$$


represents the isomorphism classes of holomorphic D-line bundles on $X$. Note that the sheaf sequence $0 \rightarrow \mathcal{D} \stackrel{\exp }{\longrightarrow} \mathcal{D}^{+} \rightarrow 1$ is exact, and therefore $H^{1}(X, \mathcal{D}) \cong H^{1}\left(X, \mathcal{D}^{+}\right)$. Now in local null coordinates we have $\mathcal{D}=e \cdot \mathcal{E}_{u}+\bar{e} \cdot \mathcal{E}_{v} \cong \mathcal{E}_{u} \oplus \mathcal{E}_{v}$ where $\mathcal{E}_{u}, \mathcal{E}_{v}$ are the sheaves of germs of functions of $u$ and $v$ respectively. Thus $H^{1}\left(X, \mathcal{D}^{+}\right) \cong H^{1}\left(X, \mathcal{E}_{u}\right) \oplus H^{1}\left(X, \mathcal{E}_{v}\right)$.

Example 8.5. (The Canonical Bundle). The bundle $\kappa \equiv \Lambda^{n, 0}$ of holomorphic $n$-forms has transition functions $\operatorname{det}_{\mathbf{D}}\left(\frac{\partial z^{\alpha}}{\partial z^{\beta}}\right)$ which are holomorphic with values in $\mathbf{D}^{+}$. In local $z$-coordinates a holomorphic $n$-form can be written as $\Phi=F(z) d z=F(z) d z_{1} \wedge \cdots \wedge d z_{n}$ where $F$ is a $\mathbf{D}$ holomorphic function. In null coordinates one has

$$
\Phi=e f(u) d u+\bar{e} g(v) d v
$$

From this we see that on a $\mathbf{D}$-manifold which is a product $X=M_{1} \times M_{2}$ as above, there always exists a global holomorphic $n$-form which is nowhere-null, i.e., $\Phi \wedge \bar{\Phi}$ is never zero. (For elements of $\Lambda^{n, 0} \mathbf{D}^{n}$ the concepts of null and non-null make sense without a metric).

Remark 8.6. (Holomorphic D-Vector Bundles). There is also the notion of a holomorphic D-vector bundle of higher rank $m$ where one has local trivializations $U_{\alpha} \times \mathbf{D}^{m}$ and transition functions which are holomophic maps to $\mathrm{GL}_{m}^{+}(\mathbf{D}) \equiv\left\{A \in \mathbf{M}_{m, m}(\mathbf{D}): \operatorname{det}_{\mathbf{D}}(A) \in \mathbf{D}^{+}\right\}$. Note that one can twist the Dolbeault complex by such bundles.

\section{Hermitian D-Manifolds.}

Let $X$ be a D-manifold and let $T X=N^{+} \oplus N^{-}$be the decomposition into the tangent spaces of the two foliations, i.e, the \pm 1-eigenbundles of the endomorphism $\mathbf{T}: T X \rightarrow T X$ in (8.3).

Definition 9.1. By a hermitian metric on $X$ we mean a non-degenerate $\mathbf{D}$-valued real-bilinear form $(\cdot, \cdot)$ on the fibres of $T X$ such that

$$
(V, W)=\overline{(W, V)} \quad \text { and } \quad(\mathbf{T} V, W)=\tau(V, W)=-(V, \mathbf{T} W)
$$

for all $V, W \in T_{x} X$ at each point $x$.

Note that

$$
(\mathbf{T} V, \mathbf{T} W)=-(V, W)
$$

from which it follows that

$$
N^{+} \text {and } N^{-} \text {are null spaces for }(\cdot, \cdot) \text {. }
$$

Recall (3.3) which expresses $(\cdot, \cdot)$ in terms of its real and imaginary parts:

$$
(V, W)=\langle V, W\rangle-\tau \omega(V, W)
$$

and note that $\langle V, W\rangle=\langle W, V\rangle, \omega(V, W)=-\omega(W, V)$ and

$$
\langle V, \mathbf{T} W\rangle=\omega(V, W) .
$$

Thus $\langle V, W\rangle$ is a semi-riemannian metric of type $(n, n)$. The spaces $N^{ \pm}$are null for this metric and for its associated "Kähler" form $\omega$. Both of these forms are characterized by the same bundle isomorphism:

$$
A: N^{+} \stackrel{\cong}{\longrightarrow}\left(N^{-}\right)^{*}
$$


Writing $V=\left(V^{+}, V^{-}\right)$with respect to the decomposition $T X=N^{+} \oplus N^{-}$, we have $\langle V, W\rangle=$ $A\left(V^{+}\right)\left(W^{-}\right)+A\left(W^{+}\right)\left(V^{-}\right)$and $\omega(V, W)=A\left(V^{+}\right)\left(W^{-}\right)-A\left(W^{+}\right)\left(V^{-}\right)$. In terms of local $z-$ coordinates and local null coordinates $(u, v)$ one has

$$
\begin{aligned}
d s^{2} & =\sum_{j, k=1}^{n} a_{j k} d z_{j} \otimes d \bar{z}_{k}=\sum_{j, k=1}^{n} \tilde{a}_{j k} d u_{j} \circ d v_{k}, \quad \text { and } \\
\omega & =\sum_{j, k=1}^{n} a_{j k} d z_{j} \wedge d \bar{z}_{k}=\sum_{j, k=1}^{n} \tilde{a}_{j k} d u_{j} \wedge d v_{k}
\end{aligned}
$$

Note 9.2. The isomorphism (9.4) shows that the existence of a D-hermitian metric on $X$ puts further topological restrictions on the bundle $T X$ (as opposed to the complex hermitian case). For example, any product $X=M_{+} \times M_{-}$of two $n$-manifolds is a $\mathbf{D}$-manifold, but (9.4) shows that a hermitian metric exists on $X$ if and only if $M_{+}$and $M_{-}$are both parallelizable. Also on any manifold $X$ the condition $T X \cong N \oplus N$ is restrictive.

Note 9.3. A D-submanifold $Y \subset X$ of a hermitian $\mathbf{D}$-manifold may not be hermitian in the induced metric. One needs the additional hypothesis that $Y$ is a symplectic submanifold, i.e., that $\left.\omega\right|_{Y}$ is non-degenerate on $Y$.

Note 9.4. On any hermitian D-manifold there exists the canonical Levi-Civita connection for the semi-riemannian metric. There also exists a canonical hermitian connection, characterized by the fact that the metric and $\mathbf{T}$ are parallel and $\nabla^{0,1}=\bar{\partial}$.

Remark 9.5. In parallel with the complex case, canonical connections exist on any holomorphic D-bundle equipped with a hermitian metric (cf. Remark 8.6). The proof follows the complex case, where one computes the connection 1-form in a holomorphic frame as $\omega=h^{-1} \partial h$ where $h$ is the matrix determined by the frame and the metric. The curvature 2 -form $\Omega=d \omega-\omega \wedge \omega$ transforms to $A \Omega A^{-1}$ under a $\mathrm{GL}_{n}(\mathbf{D})$-valued frame change $A$, so that $\operatorname{det}_{\mathbf{D}}(I+\tau \Omega)$ is a globally defined form on $X$, called the total $\mathbf{D}$-Chern form of the bundle. More generally, for any other $\mathrm{GL}_{n}(\mathbf{D})$-invariant polynomial $\phi$, the $\phi$-Chern form of the bundle, $\phi(\Omega)$, is a globally defined $d$-closed differential form. The standard transgression formula holds, and the cohomology class of $\phi(\Omega)$ in $H^{*}(X, \mathbf{D})$ is independent of the hermitian metric (or connection) on the bundle. Finally, if $h=e \cdot g+\bar{e} \cdot g^{t}$ defines a matrix $g$ with values in $\mathrm{GL}_{n}(\mathbf{R})$, then one can show that in null coordinates

$$
\tau \Omega=\tau \bar{\partial}\left(\partial h \cdot h^{-1}\right)=\bar{e} d_{u}\left(\left(d_{v} g\right) \cdot g^{-1}\right)-e d_{v}\left(\left(d_{u} g\right) \cdot g^{-1}\right)
$$

Remark 9.6. Suppose $X$ carries a nowhere-null holomorphic $n$-form $\Phi$ (see Example 8.4). Then changing the metric by the conformal factor $\|\Phi\|^{\frac{1}{n}}$ gives a new hermitian metric in which $\Phi$ has constant length. If $\Phi^{\prime}$ is another holomorphic $n$-form with constant length in this new metric, then $\Phi^{\prime}=\alpha \Phi$ for $\alpha \in \mathbf{D}$ with $\alpha \bar{\alpha} \neq 0$.

\section{Kähler D-Manifolds.}

We now consider the following natural class of hermitian double manifolds.

Definition 10.1. A hermitian D-manifold is said to be Kähler if the form $\omega$ is $d$-closed.

Interestingly, all the standard characterizations of complex Kähler manifolds carry over to this context. For example, we have the following. 
Proposition 10.2. A hermitian D-manifold $X$ is Kähler if and only if the canonical hermitian connection on $T X$ agrees with the Levi-Civita connection of the semi-riemannian metric

Proposition 10.3. Let $X$ be a hermitian D-manifold with Kähler form $\omega$ and structure map $\mathbf{T}$. Then $X$ is Káhler if and only if $\mathbf{T}$ is parallel in the Levi-Civita connection.

In the last assertion one does not need to assume apriori that the subbundles $N^{ \pm}$are integrable. The hypothesis $\nabla \mathbf{T}=0$ implies that $N^{ \pm}$are integrable.

Note 10.4. In the null-coordinate approach, a Kähler D-manifold is simply a symplectic manifold with a pair of transverse Lagrangian foliations. For this reason Kähler D-manifolds are sometimes referred to in the literature as bi-Lagrangian manifolds.

Note 10.5. Any symplectic D-submanifold of a Kähler D-manifold, is Kähler in the induced metric.

Example 10.6.(Surfaces). When $n=1$ a Kähler D-manifold is simply a surface equipped with a pair of 1 -forms $\alpha$ and $\beta$ such that $\alpha \wedge \beta$ is nowhere vanishing. Here the metric is $d s^{2}=\alpha \circ \beta$ and the Kähler form is $\omega=\alpha \wedge \beta$. Equivalently it is a Lorentzian surface.

\section{Ricci-Flat Kähler D-Manifolds.}

Let $X$ be a hermitian $\mathbf{D}$-manifold and suppose $\Phi$ is a nowhere-null holomorphic section of the canonical bundle $\kappa=\Lambda^{n, 0}(X)$. Then the real 2 -form

$$
\Omega \equiv \tau \partial \bar{\partial} \log \|\Phi\|
$$

is independent of $\Phi$. To see this note first that in local holomorphic coordinates $z$ we have $\Phi=$ $a(z) d z_{1} \wedge \ldots \wedge d z_{n}$, where $a(z)$ is a $\mathbf{D}$-holomorphic function, and $\|\Phi\|^{2}=\lambda(z) a(z) \bar{a}(z)$ where $\lambda>0$. Hence we have $\tau \partial \bar{\partial} \log \|\Phi\|^{2}=\tau \partial \bar{\partial} \log \lambda$. Changing coordinates to $z^{\prime}$ gives a new metric coefficient $\lambda^{\prime}=\lambda F \bar{F}$ where $F$ is a holomorphic function (the determinant of $\partial z / \partial z^{\prime}$ ). Thus $\partial \bar{\partial} \log \lambda=\partial \bar{\partial} \log \lambda^{\prime}$. This form $\Omega$ is the curvature of the canonical hermitian connection on $\kappa$. (This generalizes to any hermitian holomorphic line bundle.)

We observed in Remark 9.6 that given $X$ and $\Phi$ as above, there is a conformally equivalent metric on $X$ for which $\|\Phi\| \equiv 1$. Evidently, in this metric the curvature $\Omega \equiv 0$.

As remarked in (9.4), if one passes to null coordinates, then $\Phi=e f(u) d u+\bar{e} g(v) d v$. For this reason $\mathbf{D}$ geometry plays an important role in the mass-transport problem.

When $X$ is Kähler we have the following.

Proposition 11.1. Let $X$ be a Kähler D-manifold, and $\Omega$ the curvature 2-form of the canonical line bundle as above. Then

$$
\Omega(V, W)=-\operatorname{Ric}(V, \mathbf{T} W)
$$

where Ric is the Ricci curvature tensor of the Levi-Civita connection on TX. In particular, the canonical bundle is flat if and only if the Ricci curvature of $X$ is zero.

Proof. The argument goes precisely as in the complex case, with $i$ replaced by $\tau$ and the almost complex structure $J$ replaced by the structure map $\mathbf{T}$.

Thus a Ricci-flat Kähler D-manifold is the exact analogue of a Calabi-Yau manifold. The holonomy will lie in the group $\mathrm{SU}_{n}(\mathbf{D})$ (cf. [B].)

These manifolds can be characterized, in analogy with Hitchin's $\left[\mathrm{Hi}_{1}\right]$ description of the complex case, as follows. 
Proposition 11.2. A Ricci-flat Kähler D-manifold is equivalent to the data of a symplectic $2 n$-dimensional manifold $(X, \omega)$ together with two $d$-closed real $n$-forms $\phi$, $\psi$ such that:

(1) $\Phi=\phi+\tau \psi$ is a simple (indecomposable) $\mathbf{D}$-valued $n$-form.

(2) $\Phi \wedge \omega=0$, i.e. $\phi \wedge \omega=\psi \wedge \omega=0$

$$
\Phi \wedge \bar{\Phi}=\left\{\begin{array}{rlll}
\omega^{n} & \text { for } & n & \text { even } \\
-\tau \omega^{n} & \text { for } & n & \text { odd }
\end{array}\right.
$$

This proposition can be restated as follows.

Proposition 11.2'. A Ricci-flat Kähler D-manifold is equivalent to the data of a symplectic $2 n$-dimensional manifold $(X, \omega)$ together with two $d$-closed real $n$-forms $\alpha, \beta$ such that:

(1) $\Phi=e \alpha+\bar{e} \beta$ and the real $n$-forms $\alpha$ and $\beta$ are simple.

(2) $\alpha \wedge \omega=\beta \wedge \omega=0$

(3) $\alpha \wedge \beta=\omega^{n}$.

To see the equivalence of these statements, set $\alpha=\phi-\psi$ and $\beta=\phi+\psi$ and note that $\left(\alpha_{1} \wedge \cdots \wedge \alpha_{n}\right) \cdot e+\left(\beta_{1} \wedge \cdots \wedge \beta_{n}\right) \cdot \bar{e}=\left(\alpha_{1} \cdot e+\beta_{1} \cdot \bar{e}\right) \wedge \cdots \wedge\left(\alpha_{n} \cdot e+\beta_{n} \cdot \bar{e}\right)$

There are several interesting examples.

Example 11.3. By Proposition 11.2' a Ricci-flat Kähler D-manifold of (real) dimension 2 is simply a surface $\Sigma$ equipped with a pair of closed 1 -forms $\alpha, \beta$ such that $\alpha \wedge \beta$ never vanishes. (The Kähler form is $\omega=\alpha \wedge \beta$.)

Note that given this data, $\xi \equiv \alpha+i \beta$ defines a conformal structure on $\Sigma$ in which $\xi$ is a (complex) holomorphic 1-form. Conversely if $\Sigma$ is a Riemann surface with a nowhere vanishing holomorphic 1-form $\xi=\alpha+i \beta$, then $(\Sigma, \alpha, \beta)$ is a Ricci-flat Kähler D-manifold. This leads to the following.

Proposition 11.4. Every non-compact connected surface carries a Ricci-flat Kähler D-structure. The only compact connected surface to carry such a structure is the torus.

Proof. Any surface $\Sigma$ can be given a conformal structure. If $\Sigma$ is a non-compact connected Riemann surface, then it carries a nowhere vanishing holomorphic 1-form since it is Stein. If $\Sigma$ is compact, it carries such a 1 -form if and only if its genus is zero.

Since products of Ricci-flat Kähler D-manifolds are again of this type, we obtain examples with large fundamental groups in all dimensions.

Example 11.5. Let $\Sigma, \Sigma^{\prime}$ be Riemann surfaces endowed with nowhere-vanishing holomorphic 1 -forms

$$
\xi=a+i b \quad \text { and } \quad \xi^{\prime}=a^{\prime}+i b^{\prime} .
$$

On $X=\Sigma \times \Sigma^{\prime}$ we have the symplectic form and metric given by

$$
\omega=a \wedge a^{\prime}+b \wedge b^{\prime} \quad \text { and } \quad d s^{2}=a \circ a^{\prime}+b \circ b^{\prime}
$$

The tensor $\mathbf{T}: T\left(\Sigma \times \Sigma^{\prime}\right) \rightarrow T\left(\Sigma \times \Sigma^{\prime}\right)$ is defined by $\mathbf{T}=\left(\begin{array}{cc}I & 0 \\ 0 & -I\end{array}\right)$. The holomorphic 1-form

$$
\Phi=e \cdot a \wedge b+\bar{e} \cdot a^{\prime} \wedge b^{\prime} \equiv e \cdot \alpha+\bar{e} \cdot \beta
$$


satisfies conditions (1), (2) and (3) above, and so $X$ is a Ricci-flat Kähler D-manifold. One can also take products of such 4-manifolds.

Remark 11.6. (The Calabi Question). Suppose $(X, \omega)$ is a Kähler D-manifold with trivial canonical bundle. It is natural to ask the Calabi Question: Does there exist a Kähler form $\omega^{\prime}$ on the D-manifold $X$, which is cohomologous to $\omega$ and is Ricci-flat? Interestingly, the answer is: Not always. Consider the $\mathbf{D}$-structure on the torus $S^{1} \times S^{1}$ given by the transverse foliations $\mathcal{F}_{1}$ and $\mathcal{F}_{2}$, where $\mathcal{F}_{1}$ consists of the circles $S^{1} \times\{\theta\}$ for $\theta \in S^{1}$, and $\mathcal{F}_{2}$ has exactly one compact leaf $L=\left\{\theta_{0}\right\} \times S^{1}$ (whose complement is foliated by leaves which spiral barber-pole fashion from one side of $L$ to the other). Any choice of nowhere vanishing 1-forms $\alpha$ and $\beta$ so that $\alpha$ vanishes on $\mathcal{F}_{1}$ and $\beta$ vanishes on $\mathcal{F}_{2}$ makes this a Kähler $\mathbf{D}$-manifold. However, it is not possible to choose $\alpha$ and $\beta$ to be closed, that is, A Ricci-flat Kähler structure does not exist. To see this, suppose $\beta$ were closed, and consider a thin strip $S \equiv\left(\theta_{0}-\epsilon, \theta_{0}+\epsilon\right) \times S^{1}$ about the compact leaf $L$. Since $\left.\beta\right|_{L}=0$, we conclude that $\left.\beta\right|_{S}$ is exact by the deRham Theorem. That is, $\beta=d f$ where $f: S \rightarrow \mathbf{R}$ is a function whose level sets are the leaves of $\mathcal{F}_{2}$ in $S$. However, since $d f=\beta \neq 0$ on $S$, one sees that the level sets of $f$ near $L$ must be compact (circles).

Taking products gives examples in all dimensions.

\section{Split SLAG Submanifolds in the General Setting}

Let $X$ be a Hermitian $\mathbf{D}$-manifold of $\mathbf{D}$-dimension $n$ with a nowhere null holomorphic $n$-form $\Phi$. Let $\omega$ be the Kähler form of the hermitian metric. (We do not require $d \omega=0$.)

Definition 12.1. An real oriented $C^{1}$ submanifold $M$ of dimension $n$ in $X$ is said to be split SLAG (or STAG) if

(1) $M$ is Lagrangian, i.e., $\left.\omega\right|_{M}=0$.

(2) $\left.\operatorname{Im} \Phi\right|_{M}=0$.

(3) $M$ is positive space-like.

THEOREM 12.2. Suppose $\|\Phi\| \equiv 1$ on $X$. Then any compact split SLAG submanifold with boundary $(M, \partial M)$ in $X$ is homologically volume-maximizing, i.e.,

$$
\operatorname{vol}(M) \geq \operatorname{vol}(N)
$$

for any other positive space-like submanifold $N$ such that $\partial N=\partial M$ and $M-N$ is homologous to zero in $X$.

Equality holds if and only if $N$ is also split SLAG.

Proof. The same as the proof of Theorem 5.3.

Remark 12.3. One can always change the metric on $X$ by a conformal factor so that $\|\Phi\| \equiv 1$.

Remark 12.4. Theorem 12.2 carries over to split SLAG subvarieties. See Appendix D.

\section{Deformations and the McLean Theorem in the Split Case.}

In his Duke University thesis $\left[\mathrm{Mc}_{1,2}\right]$, R. McLean proved that the moduli space of Special Lagrangian submanifolds of a Ricci-flat Kähler manifold is smooth, and its tangent space at a point (submanifold) $M$ is canonically identified with the space $\mathbf{H}^{1} \cong H^{1}(M ; \mathbf{R})$ of harmonic 1forms on $M$. This result carries over to the split Special Lagrangian case (see Warren[ $\left.\mathrm{W}_{2}\right]$ ). We present this result and sketch the proof based in part on the notes of Hitchin $\left[\mathrm{Hi}_{1}\right]$. 
THEOREM 13.1. Let $X, \omega, \Phi$ be as in $\S 12$ and assume $d \omega=0$ (so $X$ is a Ricci-flat Kähler D-manifold). Let $M \subset X$ be a split SLAG submanifold, and suppose $M_{t},-\epsilon<t<\epsilon$, is a variation through split SLAG submanifolds with $M=M_{0}$. Then the corresponding normal variational vector field $\nu$ canonically gives a harmonic 1-form $\theta$ on $M$ by setting

$$
\theta=\nu \_l \omega
$$

If $M$ is compact, then, in a neighborhood of $M$, the moduli space $\mathfrak{M}$ of split SLAG submanifolds of $X$ is a manifold of dimension $b_{1}(M)$ whose tangent space at $M^{\prime} \in \mathfrak{M}$ is canonically identified with the space of harmonic 1-forms on $M^{\prime}$. In particular, this endows $\mathfrak{M}$ with a natural riemannian metric.

Proof. Let $F: M \times(-\epsilon, \epsilon) \rightarrow X$ be a smooth variation of $M=M_{0}$ so that $M_{t} \equiv F(M \times\{t\})$ is positive space-like for all $t$. Then by Definition 12.1, this is a variation through split SLAG submanifolds if and only if

$$
\left.F^{*} \omega\right|_{M \times\{t\}}=0 \quad \text { and }\left.\quad F^{*}(\operatorname{Im} \Phi)\right|_{M \times\{t\}}=0 \text { for all } t
$$

or equivalently

$$
F^{*} \omega=\widetilde{\theta} \wedge d t \quad \text { and } \quad F^{*}(\operatorname{Im} \Phi)=\widetilde{\varphi} \wedge d t
$$

where $\tilde{\theta}$ and $\widetilde{\varphi}$ are a 1 -form and an $(n-1)$-form respectively on $M \times(-\epsilon, \epsilon)$. Note that the restrictions $\theta=\left.\widetilde{\theta}\right|_{M \times\{t\}}$ and $\varphi=\left.\widetilde{\varphi}\right|_{M \times\{t\}}$ are independent of the choice of $\widetilde{\theta}$ and $\widetilde{\varphi}$. Since $d F^{*} \omega=d F^{*}(\operatorname{Im} \Phi)=0$, these restrictions satisfy $d \theta=d \varphi=0$. Note also that $\theta$ and $\varphi$ correspond to the restriction to $M_{t}$ of the contraction of $\nu \equiv F_{*}\left(\frac{\partial}{\partial t}\right)$ with $\omega$ and $\operatorname{Im} \Phi$ respectively. In fact, by 12.1 (1) and (2), only the normal part of $F_{*}\left(\frac{\partial}{\partial t}\right)$ survives under restriction. The first part of the Theorem is now a consequence of the following algebraic assertion:

$$
\varphi=* \theta
$$

where $*$ denotes the Hodge star-operator with respect to the induced riemannian metric on $M_{t}$. The proof of (13.1) is straightforward and left to the reader.

The proof of the unobstructedness of the moduli space near $M$, when $M$ is compact, follows exactly the argument given in $\left[\mathrm{Mc}_{2}\right]$.

\section{Relation to the Mass Transport Problem - Work of Kim, McCann and Warren}

We first recall the classical Monge-Kantorovich Optimal Mass Transport Problem in a smooth setting. Fix $n$-dimensional manifolds $U$ and $V$ with domains $\Omega_{U} \subset \subset U, \Omega_{V} \subset \subset V$ and smooth positive densities $\rho_{U}, \rho_{V}$ on $\Omega_{U}$ and $\Omega_{V}$ respectively. Let $c: \Omega_{U} \times \Omega_{V} \rightarrow \mathbf{R}$ be a smooth "cost" function. Set

$$
\mathcal{M} \equiv\left\{F: \Omega_{U} \rightarrow \Omega_{V}: F_{*}\left(\rho_{U}\right)=\rho_{V}\right\}
$$

Problem. Find a mapping in $\mathcal{M}$ which minimizes the functional

$$
C(F) \equiv \int_{\Omega_{U}} c(u, F(u)) \rho_{U}
$$


It turns out that this is equivalent to the following. Consider the space

$$
\mathcal{L} \equiv\left\{(f, g) \in C\left(\Omega_{U}\right) \times C\left(\Omega_{V}\right): f(u)+g(v) \leq c(u, v) \forall u, v\right\}
$$

Dual Problem. Find a pair $(f, g) \in \mathcal{F}$ which maximizes the functional

$$
J(f, g) \equiv \int f(u) \rho_{U}+\int g(v) \rho_{V}
$$

Consider now the case $U=V=\mathbf{R}^{n}$. Under appropriate conditions on the cost function these equivalent problems have a unique solution. For the classical cost function $c(u, v)=\frac{1}{2}|u-v|^{2}$ the solutions are related by

$$
F=d f=(d g)^{-1}=F^{-1} .
$$

Note that $f$ and $g$ are Legendre transforms of one-another. If we write $\rho_{U}=\rho(u) d u$ and $\rho_{V}=$ $\widetilde{\rho}(v) d v$ for smooth positive functions $\rho, \widetilde{\rho}$, then $f$ satisfies the Monge-Ampère equation

$$
\operatorname{det} \operatorname{Hess} f=\frac{\rho}{\widetilde{\rho}(d f)} .
$$

For a complete and detailed exposition of the modern state of knowledge pertaining to the optimal transport problem, the reader is referred to [V].

Even when one is working in the general case presented above, there are existence and uniqueness results. Kim, McCann and Warren have found that this problem and its solution fit beautifully into the framework of Kähler D-manifolds. Briefly it goes as follows.

Consider the D-manifold

$$
U \times V .
$$

The first assumption is that the given cost function gives a global Kähler potential, i.e.,

$$
\omega \equiv d_{u} d_{v} c
$$

is a symplectic (i.e., non-degenerate) 2-form on $U \times V$. (Otherwise said, the matrix $\frac{\partial^{2} c}{\partial u_{i} \partial v_{j}}$ is everywhere non-singular,) This makes $U \times V$ into a Kähler D-manifold. One also imposes a so-call "twist condition" on $c$ which says that the maps $u \mapsto\left(d_{v} c\right)(u, v)$ and $v \mapsto\left(d_{u} c\right)(u, v)$ are injective, and which we can ignore here.

Observe now that our Kähler D-manifold is equipped with a holomorphic $n$-form

$$
\Phi \equiv \rho(u) d u \cdot e+\widetilde{\rho}(v) d v \cdot \bar{e}
$$

THEOREM 14.1. [KMW]. The graph of the unique solution to the Monge-Kantorovich optimal mass transport problem given above is a split SLAG submanifold of the Kähler $\mathbf{D}$-manifold $U \times V$.

Note that in general $U \times V$ is not Ricci-flat. Hence the graph of $F$ is only volume-maximizing for a conformally related metric (cf. Remark 12.3).

\section{Further Work of Mealy}

In this section we give a brief overview of the primary work on semi-riemannian calibrated geometry $\left[\mathrm{M}_{1,2}\right]$. Let $\mathbf{R}^{p, q}$ denote the standard semi-euclidean vector space of signature $p, q \geq 1$. 
Choose a connected component $G$ of the grassmannian of oriented non-degenerate linear subspaces of $\mathbf{R}^{p, q}$ of dimension $k=r+s$ and signature $r, s$. There is a canonical embedding of $G$ into $\Lambda^{r+s} \mathbf{R}^{p, q}$ via the wedge product of any oriented orthonormal basis for the subspace.

Definition 15.1. Given a subset $A \subset G$, a form $\phi \in \Lambda^{r+s}\left(\mathbf{R}^{p, q}\right)^{*}$ is a calibration of type $A$ if

$$
\phi(\xi) \geq 1 \text { for all } \xi \in A \text {. }
$$

Moreover, the set

$$
G(\phi)=\{\xi \in A: \phi(\xi)=1\}
$$

of calibrated planes is usually assumed to be non-empty. An oriented submanifold $M$ is of type $A$ if each tangent plane is in $A$. We say $M$ is calibrated by $\phi$ if $M$ is of type $G(\phi)$.

The cases where $A$ can be taken to be a full connected component of $G$ are very limited. Ignoring the two cases of dimension $k=0$ and dimension $k=n$, where $\phi$ is \pm the volume form on $\mathbf{R}^{p, q}$, and eliminating duplications from interchanging $p$ and $q$, Mealy shows that only two cases (essentially one) remain. The Grassmannian $G_{\text {space }}\left(p, \mathbf{R}^{p, q}\right)$ of maximally space-like oriented planes in $\mathbf{R}^{p, q}$, has two connected components. Let $G_{\text {space }}^{+}\left(p, \mathbf{R}^{p, q}\right)$ denote the component containing $\overrightarrow{\mathbf{R}}^{p}=\mathbf{R}^{p} \times\{0\}$ with its standard orientation. The other component is $G_{\text {space }}^{-}\left(p, \mathbf{R}^{p, q}\right)=$ $-G_{\text {space }}^{+}\left(p, \mathbf{R}^{p, q}\right)$. Henceforth we only consider $G_{\text {space }}^{+}\left(p, \mathbf{R}^{p, q}\right)$. Thus the problem of finding semiriemannian calibrations $\phi$ of type $A$, where $A$ is a connected component of the non-degenerate Grassmannian, is reduced to the case $A \equiv G_{\text {space }}^{+}\left(p, \mathbf{R}^{p, q}\right)$ and the problem of finding a form

$$
\phi \in \Lambda^{r+s}\left(\mathbf{R}^{p, q}\right)^{*} \quad \text { with } \phi(\xi) \geq 1 \quad \forall \xi \in \phi \in G_{\text {space }}^{+}\left(p, \mathbf{R}^{p, q}\right) .
$$

Remark 15.2. In the next section we will give an important example of "type $A$ " where $A$ is a proper subset of a component of the Grassmannian.

THEOREM 15.3. Suppose that $\phi$ is a calibration of type $A$ and $M$ is a compact submanifold of $\mathbf{R}^{p, q}$ calibrated by $\phi$. If $N$ is any other compact submanifold of type $A$ with $\partial N=\partial M$, then

$$
\operatorname{vol}(M) \geq \operatorname{vol}(N)
$$

with equality if and only if $N$ is also calibrated by $\phi$.

Proof. See the proof of Theorem 5.3.

Example 1. (Point Calibrations). The inner product $\langle\cdot, \cdot\rangle$ of signature $p, q$ on $\mathbf{R}^{p, q}$ induces an isomorphism $\mathbf{R}^{p, q} \stackrel{\cong}{\longrightarrow}\left(\mathbf{R}^{p, q}\right)^{*}$ which extends to an equivalence between $\Lambda^{k} \mathbf{R}^{p, q}$ and $\Lambda^{k}\left(\mathbf{R}^{p, q}\right)^{*}$. Given $\xi \in G_{\text {space }}^{+}\left(p, \mathbf{R}^{p, q}\right)$, the equivalent form $\phi_{\xi} \in \Lambda^{p}\left(\mathbf{R}^{p, q}\right)^{*}$ defined by $\phi_{\xi}(\eta)=\langle\xi, \eta\rangle$ is a calibration of type $G_{\text {space }}^{+}\left(p, \mathbf{R}^{p, q}\right)$. More precisely one has:

$$
\begin{gathered}
\phi_{\xi}(\eta) \geq 1 \text { for } \eta \in G_{\text {space }}^{+}\left(p, \mathbf{R}^{p, q}\right) \\
\text { with equality } \Longleftrightarrow \eta=\xi
\end{gathered}
$$

With a conflict of terminology, the case $p=1$ yields a calibration proof of the twin paradox for curves in $\mathbf{R}^{1, q}$ by taking the first axis to be time (see [H, Prop 4.19]). The proof of the inequality above is immediate (see the bottom of page 797 in $\left[\mathrm{M}_{2}\right]$ ) from a canonical form for $\eta$ with respect to $\xi$ under the action of the orthogonal group $\mathrm{O}(p, q)$ given on page 796 of $\left[\mathrm{M}_{2}\right]$. 
Example 2. (Divided Powers of the Kähler Form). Now take $\mathbf{C}^{p, q}$ to be $\mathbf{C}^{p+q}$ equipped with the standard complex hermitian inner product of signature $p, q$, and let $\omega$ denote the corresponding "Kähler" form. In general, $\phi \equiv \frac{1}{k !} \omega^{k}$ is not a calibration. It is a calibration only when $k=p$.

Example 3. (Split Associative, Coassociative, etc.). Replacing the octonions $\mathbf{O}$ by their split companion $\widetilde{\mathbf{O}}$ (analogous to replacing $\mathbf{C}$ by its split companion $\widetilde{\mathbf{C}}=\mathbf{D}$ ), the associative, coassociative and Cayley calibrations have counterparts that are calibrations.

Finally it is worth noting that all possible calibrations of type $G_{\text {space }}^{+}\left(3, \mathbf{R}^{3,3}\right)$ are classified in $\left[\mathrm{M}_{1}\right]$. This is the lowest dimensional non-trivial case.

\section{Lagrangian Submanifolds of Constant Phase and Volume Maximization}

In this section we look at the general picture of all oriented Lagrangian submanifolds with non-degenerate metric. We show that such submanifolds are minimal (mean curvature zero) if and only if they are of constant phase. When and only when the submanifolds are also purely spacelike or time-like, they are homologically volume-maximizing among purely space-like or time-like submanifolds of the same type. However, in all other signatures they are homologically volumemaximizing among Lagrangian submanifolds of the same type. For convenience we shall work in $\mathbf{D}^{n}$, however the results hold in any Ricci-flat $\mathbf{D}$-manifold.

The first result of this nature was noted in $\left[\mathrm{HL}_{1}\right.$, page 96$]$ in the standard special Lagrangian setting. The phase $\theta$ of a Lagrangian submanifold $M$ of $\mathbf{C}^{n}$ was defined using the fact that $d z(\vec{M})=e^{i \theta}$ is of unit length (Proposition 1.14 and (2.18)). For any tangent vector $V$ to $M$ one has that $V(\theta)=\langle J V, H\rangle$ (equation (2.19)) where $H$ is the mean curvature of $M$.

Dong [D] considered the case of non-degenerate Lagrangian submanifolds $M$ of $\mathbf{C}^{p, q}\left(\mathbf{C}^{p+q}\right.$ with the standard hermitian inner product of signature $p, q)$. Again the phase $\theta$ can be defined by $e^{i \theta}=(d z)(\vec{M})$ since it is of unit length. Despite the fact that Re $d z$ is not a calibration, Dong noted that $V(\theta)=\langle J V, H\rangle$ still holds, so that $M$ is of mean curvature zero if and only if the phase function is constant ([D, Lemma 2.1]). Each non-degenerate Lagrangian subspace in $\mathbf{C}^{p, q}$ must have signature $p, q$. We would like to make the further observation that Re $d z$, although not a calibration in the pure sense, is a calibration of type $A=\mathrm{LAG}$, the set of oriented non-degenerate Lagrangian subspaces, because $(d z)(\xi)=e^{i \theta}(\xi)$ for $\xi \in$ LAG. By arguing as in the proof of Theorem 15.4, this proves that a constant phase Lagrangian submanifold is volume-minimizing among all other Lagrangian submanifold with the same boundary.

We now turn attention back to $\mathbf{D}^{n}$. Let LAG denote the set of oriented non-degenerate Lagrangian $n$-planes in $\mathbf{D}^{n}$. The set LAG decomposes into $2 n+2$ connected components

$$
\mathrm{LAG}=\coprod_{p+q=n} \mathrm{LAG}_{p, q}^{ \pm}
$$

where $\mathrm{LAG}_{p, q}^{ \pm}$consists of planes for which the induced metric has signature $(p, q)$ and orientation + or - when compared to an a priori chosen model. Each $\mathrm{LAG}_{p, q}^{+}$and $\mathrm{LAG}_{p, q}^{-}$is an orbit of the group $\mathrm{U}_{n}^{+}(\mathbf{D})$, and the pair $\mathrm{LAG}_{p, q}^{+} \cup \mathrm{LAG}_{p, q}^{-}$is an orbit of $\mathrm{U}_{n}(\mathbf{D})$.

Note 16.1. The oriented model planes can be chosen so that

$$
\pm\left.\tau^{q} d z\right|_{P}=e^{\tau \theta_{P}} d \operatorname{vol}_{P} \quad \text { for } P \in \mathrm{LAG}_{p, q}^{ \pm} \text {. }
$$

where $\theta_{P} \in \mathbf{R}$ and $d \operatorname{vol}_{P}$ is the unit (positive) volume form on $P$. 
If $M$ is an oriented connected non-degenerate Lagrangian submanifold of signature $p, q$, then all its tangent planes lie either in $\mathrm{LAG}_{p, q}^{+}$or in $\mathrm{LAG}_{p, q}^{-}$depending on the orientation of $M$ and we say that $M$ is of type $\mathrm{LAG}_{p, q}^{+}$or of type $\mathrm{LAG}_{p, q}^{-}$. Therefore by (16.1) we have that

$$
\pm\left.\tau^{q} d z\right|_{M}=e^{\tau \theta} d \operatorname{vol}_{M}
$$

Definition 16.2. This smooth real-valued function $\theta$ on $M$ will be called the phase function on $M$. If $\theta$ is constant, we say that $M$ has constant phase.

Proposition 16.3. Let $M \subset \mathbf{D}^{n}$ be an oriented non-degenerate Lagrangian submanifold which is connected. Then $M$ is a minimal (mean curvature zero) submanifold if and only if $M$ has constant phase.

Proof. This follows immediately from the fact that for any tangent vector field $V$ on $M$

$$
V \cdot \theta=\langle\mathbf{T} V, H\rangle
$$

where $H$ is the mean curvature vector of $M$. By (9.3) this is equivalent to the statement that

$$
d \theta=H_{-} l \omega
$$

A proof of (16.3) is given at the end of the section.

Proposition 16.4. The form

$$
\phi_{\theta}= \pm \operatorname{Re}\left\{e^{-\tau \theta} \tau^{q} d z\right\}
$$

is a calibration of type $\mathrm{LAG}_{p, q}^{ \pm}$.

Proof. Apply (16.1) and (16.2).

As noted in Theorem 5.3 and Remark 5.4, if $M$ is a compact submanifold (with boundary) in $\mathbf{D}^{n}$ of type $\mathrm{LAG}_{n, 0}^{+}$which has constant phase $\theta$, then for any submanifold $N$ of type $G_{\text {space }}^{+}\left(n, \mathbf{D}^{n}\right)$ with the same boundary as $M$ we have $\operatorname{vol}(M) \geq \operatorname{vol}(N)$, and equality holds if and only if $N$ is also of type $\mathrm{LAG}_{n, 0}^{+}$with the same constant phase. A similar result holds for $\mathrm{LAG}_{0, n}^{+}$(and of course for $\mathrm{LAG}_{n, 0}^{-}$), but not for $\mathrm{LAG}_{p, q}^{+}$if $p, q \geq 1$ unless one restricts attention of a subset of the appropriate Grassmannian (See Remark 15.2).

THEOREM 16.5. Suppose that $(M, \partial M)$ is a compact submanifold of type $\mathrm{LAG}_{p, q}^{ \pm}$in $\mathbf{D}^{n}$ which is of constant phase $\theta$. If $(N, \partial N)$ is any other compact submanifold of the same type $\mathrm{LAG}_{p, q}^{ \pm}$with $\partial N=\partial M$, then

$$
\operatorname{vol}(M) \geq \operatorname{vol}(N)
$$

with equality if and only if $N$ is also of constant phase $\theta$.

Proof. Apply Theorem 15.3 and Proposition 16.4.

Remark 16.6. This theorem carries over to any Ricci-flat Kähler D-manifold. Here $M$ is only homologically volume maximizing, i.e., maximizing among $M^{\prime}$ where $M-M^{\prime}=\partial Y$ for some $(n+1)$-chain $Y$.

Proof of formula (16.3) Pick a local oriented orthonormal frame field $e_{1}, \ldots, e_{n}$ on $M$. Then

$$
d z\left(e_{1} \wedge \cdots \wedge e_{n}\right)=e^{\tau \theta}
$$


defines the smooth phase function $\theta$ for $M$. Obviously we have that $V(\theta)=\tau e^{-\tau \theta} V\left(e^{\tau \theta}\right)=$ $\tau e^{-\tau \theta} V\left(d z\left(e_{1} \wedge \cdots \wedge e_{n}\right)\right)$. Now with $\nabla$ the riemannian connection on $M$, we have

$$
\begin{aligned}
V\left(d z\left(e_{1} \wedge \cdots \wedge e_{n}\right)\right) & =d z\left(\sum_{k=1}^{n} e_{1} \wedge \cdots \wedge \nabla_{V} e_{k} \wedge \cdots \wedge e_{n}\right) \\
& =d z\left(\sum_{k=1}^{n} e_{1} \wedge \cdots \wedge\left\langle\nabla_{V} e_{k}, \mathbf{T} e_{k}\right\rangle \mathbf{T} e_{k} \wedge \cdots \wedge e_{n}\right)=\sum_{k=1}^{n}\left\langle\nabla_{V} e_{k}, \mathbf{T} e_{k}\right\rangle \tau e^{\tau \theta}
\end{aligned}
$$

first because $\nabla d z=0$, second because $\left\langle\nabla e_{k}, e_{k}\right\rangle=0$ and $d z\left(e_{\ell} \wedge \mathbf{T} e_{\ell} \wedge \eta\right)=0$, with the third equality following because $d z\left(e_{1} \wedge \cdots \wedge \mathbf{T} e_{k} \wedge \cdots \wedge e_{n}\right)=\tau d z\left(e_{1} \wedge \cdots \wedge e_{n}\right)=\tau e^{\tau \theta}$. Combining gives

$$
V(\theta)=\sum_{k=1}^{n}\left\langle\nabla_{V} e_{k}, \mathbf{T} e_{k}\right\rangle,
$$

but $\left\langle\nabla_{V} e_{k}, \mathbf{T} e_{k}\right\rangle=\left\langle\nabla_{e_{k}} V, \mathbf{T} e_{k}\right\rangle=-\left\langle\nabla_{e_{k}} \mathbf{T} V, e_{k}\right\rangle=\left\langle\mathbf{T} V, \nabla_{e_{k}} e_{k}\right\rangle$ and $H=\sum_{k}\left(\nabla_{e_{k}} e_{k}\right)^{\text {normal }}$ is the mean curvature.

\section{Appendix A}

\section{A Canonical Form for Space-like n-Planes in $\mathrm{D}^{n}$.}

In this appendix we give a canonical form for space-like $n$-planes under the unitary group. We first treat the case $n=2$ which is the key.

Lemma A.1. Given a space-like 2-plane $P$ in $\mathbf{D}^{2}$, there exist a space-like $\mathbf{D}$-unitary basis $e_{1}, e_{2}$ for $\mathbf{D}^{2}$ and an angle $\theta \geq 0$ such that $e_{1}$ and $\cosh \theta e_{2}+\sinh \theta \mathbf{T} e_{1}$ form an orthonormal basis for $P$.

Proof. Pick any unit vector $e_{1} \in P$. Since $P$ is space-like, there exists a unit time-like vector $\mathbf{T} e_{2}$ orthogonal to both $P$ and $\mathbf{T} e_{1}$. Let this define the unit space-like vector $e_{2}$. Then the unit vector $\epsilon \in P$ orthogonal to $e_{1}$ belongs to the span of $e_{2}$ and $\mathbf{T} e_{1}$. Hence $\epsilon=\cosh \theta e_{2}+\sinh \theta \mathbf{T} e_{1}$, after possible sign changes of $e_{1}$ and $e_{2}$ to ensure $\theta \geq 0$.

Note. Using the basis $e_{1}, e_{2}$ to define coordinates $z_{1}=x_{1} e_{1}+y_{1} \mathbf{T} e_{1}$ and $z_{2}=x_{2} e_{2}+y_{2} \mathbf{T} e_{2}$. The 2-plane $P$ is defined by $y_{1}=(\tanh \theta) x_{2}$ and $y_{2}=0$ (where $\left.-1<\tanh \theta<1\right)$, i.e., $P$ is the graph over $\mathbf{R}^{2}$ in $\mathbf{R}^{2} \oplus \tau \mathbf{R}^{2}$ of the nilpotent matrix $A=\left(\begin{array}{cc}0 & \tanh \theta \\ 0 & 0\end{array}\right)$.

Proposition A.2. (Canonical Form). Each space-like $n$-plane $P$ in $\mathbf{D}^{n}$ has an orthonormal basis

$$
\begin{gathered}
\left\{e_{1}, \cosh \theta_{1} e_{2}+\sinh \theta_{1} \mathbf{T} e_{1}, \ldots, e_{n-1}, \cosh \theta_{\frac{n}{2}} e_{n}+\sinh \theta_{\frac{n}{2}} \mathbf{T} e_{n-1}\right\} \quad \text { if } n \text { is even, and } \\
\left\{e_{1}, \cosh \theta_{1} e_{2}+\sinh \theta_{1} \mathbf{T} e_{1}, \ldots, e_{n-2}, \cosh \theta_{\frac{n-1}{2}} e_{n-1}+\sinh \theta_{\frac{n-1}{2}} \mathbf{T} e_{n-2}, e_{n}\right\} \quad \text { if } n \text { is odd, }
\end{gathered}
$$

where $e_{1}, \ldots, e_{n}$ is a space-like $\mathbf{D}$-unitary basis for $\mathbf{D}^{n}$ and $\theta_{1} \geq \cdots \geq \theta_{\left[\frac{n}{2}\right]} \geq 0$.

Proof. The case $n=2$ has already been proven. The 2 -form $\left.\omega\right|_{P}$ can be put in canonical form with respect to the positive definite inner product $\langle\cdot, \cdot\rangle$ on $P$. (This is the same as the canonical 
form for a skew $n \times n$-matrix under the conjugate action of the orthogonal group.) Namely, there exist an orthonormal basisi $\epsilon_{1}, \ldots, \epsilon_{n}$ for $P$ and $\lambda_{1} \geq \cdots \geq \lambda_{\left[\frac{n}{2}\right]} \geq 0$ such that:

$$
\left.\omega\right|_{P}=\sum_{j=1}^{\left[\frac{n}{2}\right]} \lambda_{j} \epsilon_{2 j-1} \wedge \epsilon_{2 j} .
$$

Note that $\operatorname{span}\left\{\epsilon_{1}, \epsilon_{2}, \mathbf{T} \epsilon_{1}, \mathbf{T} \epsilon_{2}\right\} \cong \mathbf{D}^{2}$ and $\operatorname{span}\left\{\epsilon_{3}, \epsilon_{4}, \mathbf{T} \epsilon_{3}, \mathbf{T} \epsilon_{4}\right\} \cong \mathbf{D}^{2}$ are orthogonal in $\mathbf{D}^{n}$ since $\left\langle\epsilon_{i}, \mathbf{T} \epsilon_{j}\right\rangle=\omega\left(\epsilon_{i}, \epsilon_{j}\right)$. Thus we can apply induction.

Next we keep track of orientations. Let $\xi_{0}=\overrightarrow{\mathbf{R}}^{n}$ denote the space-like $n$-plane $\mathbf{R}^{n} \subset \mathbf{D}^{n}=$ $\mathbf{R}^{n} \oplus \tau \mathbf{R}^{n}$ equipped with the standard orientation. Let $G_{\text {space }}^{+}\left(n, \mathbf{D}^{n}\right)$ denote the connected component of the Grassmannian of oriented space-like $n$-planes in $\mathbf{D}^{n}$ which contains $\xi_{0}$. Given $\xi \in G_{\text {space }}^{+}\left(n, \mathbf{D}^{n}\right)$ the orthonormal basis $\epsilon_{1}, \ldots, \epsilon_{n}$ for $P$, described in Proposition A.2, satisfies $\xi= \pm \epsilon_{1} \wedge \cdots \wedge \epsilon_{n}$. If $n$ is odd and the minus sign occurs, we can replace $e_{n}=\epsilon_{n}$ by its negative and have a canonical oriented basis. If $n$ is even and the minus sign occurs, replace $e_{n}$ and $\theta_{\left[\frac{n}{2}\right]}$ by their negatives. This replaces $\epsilon_{n}=\cosh \theta_{\frac{n}{2}} e_{n}+\sinh \theta_{\frac{n}{2}} \mathbf{T} e_{n-1}$ by its negative, and we have proved the following oriented version of the canonical form.

Proposition A.3 (Canonical Form - Oriented Version). Each $\xi \in G_{\text {space }}^{+}\left(n, \mathbf{D}^{n}\right)$ can be put in the canonical form

$\xi=e_{1} \wedge\left(\cosh \theta_{1} e_{2}+\sinh \theta_{1} \mathbf{T} e_{1}\right) \wedge \cdots \wedge e_{n-1} \wedge\left(\cosh \theta_{\frac{n}{2}} e_{n}+\sinh \theta_{\frac{n}{2}} \mathbf{T} e_{n-1}\right) \quad$ if $n$ is even, and $\xi=e_{1} \wedge\left(\cosh \theta_{1} e_{2}+\sinh \theta_{1} \mathbf{T} e_{1}\right) \wedge \cdots \wedge e_{n-2} \wedge\left(\cosh \theta_{\frac{n-1}{2}} e_{n-1}+\sinh \theta_{\frac{n-1}{2}} \mathbf{T} e_{n-2}\right) \wedge e_{n} \quad$ if $n$ odd where $e_{1}, \ldots, e_{n}$ is a space-like $\mathbf{D}$-unitary basis for $\mathbf{D}^{n}$ and $\theta_{1} \geq \cdots \geq \theta_{\left[\frac{n}{2}\right]}$; all $\geq 0$ if $n$ is odd, and all but one can be taken $\geq 0$ if $n$ is even. Moreover, $e_{1} \wedge \cdots \wedge e_{n} \in G_{\text {space }}^{+}\left(n, \mathbf{D}^{n}\right)$. Consequently, the map $A \in M_{n}(\mathbf{D})$ sending the standard space-like unitary basis $\mathbf{e}_{1}, \ldots, \mathbf{e}_{n}$ for $\mathbf{D}^{n}$ to the space-like unitary basis $e_{1}, \ldots, e_{n}$ for $\mathbf{D}^{n}$ has $\operatorname{det}_{\mathbf{D}} A=r e^{\tau \theta}, r>0$, that is, $A \in \mathrm{U}_{n}^{+}(\mathbf{D})$.

Proof. The only thing left to prove is that $e_{1} \wedge \cdots \wedge e_{n} \in G_{\text {space }}^{+}\left(n, \mathbf{D}^{n}\right)$. The homothety connecting $\theta$ to 0 connects $\xi$ to $e_{1} \wedge \cdots \wedge e_{n}$. Since $\xi \in G_{\text {space }}^{+}\left(n, \mathbf{D}^{n}\right)$, this proves that $e_{1} \wedge \cdots \wedge e_{n} \in G_{\text {space }}^{+}\left(n, \mathbf{D}^{n}\right)$.

\section{Appendix B}

\section{Projective and Hermitian Projective varieties over D.}

Much of hermitian projective geometry carries over to the $\mathbf{D}$ setting, with some interesting twists. We begin with the following.

Definition B.1. The $n$-dimensional projective space $\mathbf{P}^{n}(\mathbf{D})$ over $\mathbf{D}$ is the set of all free rankone submodules of $\mathbf{D}^{n+1}$. Otherwise said, $\mathbf{P}^{n}(\mathbf{D})$ is the set of all $T$-invariant real 2-dimensional subspaces $\ell \subset \mathbf{D}^{n+1}$ for which the +1 and -1 eigenspaces $\ell_{ \pm}$are both one-dimensional.

Passing to null coordinates and writing $\mathbf{D}^{n+1}=e \mathbf{R}^{n+1} \oplus \bar{e} \mathbf{R}^{n+1}$ (the \pm -eigenspaces of $T$ ), we see that the decomposition $\ell=\ell_{+} \oplus \ell_{-}$gives

$$
\mathbf{P}^{n}(\mathbf{D})=\mathbf{P}^{n}(\mathbf{R}) \times \mathbf{P}^{n}(\mathbf{R})
$$


There is a corresponding decomposition for all the $\mathbf{D}$-grassmannians. In fact we have the following. For every real projective algebraic variety $V(\mathbf{R})$ there is a corresponding variety $V(\mathbf{D})$ over $\mathbf{D}$ defined by the same polynomials with arguments now in $\mathbf{D}^{n+1}$ (base change). Taking null coordinates $z=e u+\bar{e} v \in \mathbf{D}^{n+1}=e \mathbf{R}^{n+1} \oplus \bar{e} \mathbf{R}^{n+1}$, a polynomial $p(x)$ with real coefficients satisfies $p(z)=e p(u)+\bar{e} p(v)$ which shows that

$$
V(\mathbf{D})=V(\mathbf{R}) \times V(\mathbf{R}) .
$$

Note that in choosing homogeneous coordinates for $\mathbf{P}^{n}(\mathbf{D})$ one must restrict to elements $z \in \mathbf{D}^{n+1}$ which generate a free submodule. This means that $z=e u+\bar{e} v$ where $u \neq 0$ and $v \neq 0$. The space $\mathbf{P}^{n}(\mathbf{D})$ is then obtained by dividing by the group $\mathbf{D}^{*}$.

When we introduce the split Fubini-Study metric this all becomes more interesting. Recall the hermitian metric $(z, \zeta)=\sum_{k=0}^{n} z_{k} \bar{\zeta}_{k}$ with associated quadratic form

$$
(z, z)=\sum_{k=0}^{n} u_{k} v_{k} \equiv u \bullet v .
$$

In $\mathbf{P}^{n}(\mathbf{D})$ there is the projective Stiefel variety:

$$
\begin{aligned}
\mathrm{St} & \equiv\left\{[z] \in \mathbf{P}^{n}(\mathbf{D}):(z, z)=0\right\} \\
& =\left\{[e u+\bar{e} v] \in \mathbf{P}^{n}(\mathbf{D}): u \bullet v=0\right\} \\
& \cong\left\{\left(\ell_{1}, \ell_{2}\right) \in \mathbf{P}^{n}(\mathbf{R}) \times \mathbf{P}^{n}(\mathbf{R}): \ell_{1} \perp \ell_{2}\right\}
\end{aligned}
$$

which is a hypersurface (dimension $2 n-1$ ) in $\mathbf{P}^{n}(\mathbf{R}) \times \mathbf{P}^{n}(\mathbf{R})$. It is not difficult to check that $\left(\mathbf{D}^{n+1}\right)^{*} \equiv \mathbf{D}^{n+1}-\{(z, z)=0\}=\mathbf{D}^{n+1}-\{u \bullet v=0\}$ has four connected components corresponding to the four components of the unit sphere $\boldsymbol{S} \equiv\{u \bullet v=1\}$. They are permuted by the group $\{ \pm 1\} \times\{ \pm \tau\}$. We define the $\mathbf{D}$ hermitian projective space to be the connected open set

$$
\mathbf{P}_{\text {herm }}^{n}(\mathbf{D}) \equiv \mathbf{P}^{n}(\mathbf{D})-\mathrm{St}
$$

and note the mapping $\left(\mathbf{D}^{n+1}\right)^{*} \rightarrow\left(\mathbf{D}^{n+1}\right)^{*} / \mathbf{D}^{*}=\mathbf{P}_{\text {herm }}^{n}(\mathbf{D})$ obtained by dividing by the group $\mathbf{D}^{*}$. Restricting to the unit sphere gives the split Hopf mapping

$$
\mathbf{S} \longrightarrow \mathbf{P}_{\text {herm }}^{n}(\mathbf{D})
$$

with fibres which are orbits of the unit circle $S \equiv\left\{z \in \mathbf{D}^{+}:\langle z, z\rangle= \pm 1\right\}$ acting by scalar multiplication.

We point out that the group $\mathrm{GL}_{n+1}(\mathbf{D})$ acts transitively on $\mathbf{P}^{n}(\mathbf{D})$ and the group $\mathrm{U}_{n+1}(\mathbf{D})$ acts transitively on the open subset $\mathbf{P}_{\text {herm }}^{n}(\mathbf{D})$. Thus,

$$
\mathbf{P}^{n}(\mathbf{D})=\mathrm{GL}_{n+1}(\mathbf{D}) / \mathrm{GL}_{1, n}(\mathbf{D}) \quad \supset \quad \mathbf{P}_{\text {herm }}^{n}(\mathbf{D})=\mathrm{U}_{n+1}(\mathbf{D}) / \mathrm{U}_{1}(\mathbf{D}) \times \mathrm{U}_{n}(\mathbf{D})
$$

where $\mathrm{GL}_{1, n}(\mathbf{D})$ is the subgroup preserving the initial line.

Now in hermitian homogeneous coordinates $\left(\mathbf{D}^{n+1}\right)^{*}$ consider the real $\mathrm{U}_{n+1}(\mathbf{D})$-invariant 2form

$$
\widetilde{\omega} \equiv-\tau \partial \bar{\partial} \log (z, z)
$$

which in null coordinates can be written

$$
\widetilde{\omega}=d_{u} d_{v} \log (u \bullet v)=\frac{1}{u \bullet v} \sum_{j} d u_{j} \wedge d v_{j}-\frac{1}{(u \bullet v)^{2}}(v \bullet d u) \wedge(u \bullet d v) .
$$


Fix a point $\left(u_{0}, v_{0}\right) \in \mathbf{D}^{n+1}$ with $u_{0} \bullet v_{0}=1$. Then $\nu=\left(u_{0}, v_{0}\right)$, translated to $\left(u_{0}, v_{0}\right)$ is the unit normal to the sphere $\boldsymbol{S}$ at this point, and the horizontal subspace $H_{\left(u_{0}, v_{0}\right)} \subset T_{\left(u_{0}, v_{0}\right)} \boldsymbol{S}$ is the D-orthogonal complement of $\nu$. Note that $\nu_{-} l \widetilde{\omega}=u_{0} \bullet d v-v_{0} \bullet d u-\left(v_{0} \bullet u_{0}\right) u_{0} \bullet d v+\left(u_{0} \bullet v_{0}\right) v_{0} \bullet d u=$

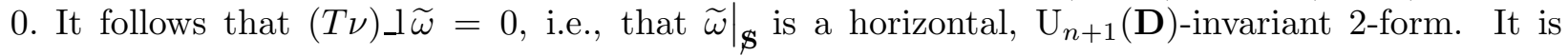
straightforward to check that $\widetilde{\omega}$ is non-degenerate on $H_{u_{0}, v_{0}}$. (It is particularly clear at points $\left(u_{0}, u_{0}\right)$. Then use the transitivity of $\mathrm{U}_{n+1}(\mathbf{D})$.) We have proved the following.

Lemma B.2. The form $\widetilde{\omega}$ descends to a $d$-closed $\mathrm{U}_{n+1}(\mathbf{D})$-invariant symplectic 2-form $\omega$ on $\mathbf{P}_{\text {herm }}^{n}(\mathbf{D})$. This form makes $\mathbf{P}_{\text {herm }}^{n}(\mathbf{D})$ a $\mathbf{D}$-Kähler manifold.

From Note 10.5 we have the following.

Corollary B.3. Let $X \subset \mathbf{P}^{n}(\mathbf{D})$ be a $\mathbf{D}$-submanifold and let $X_{\text {herm }} \subset \mathbf{P}_{\text {herm }}^{n}$ (D) denote the set of points in $X$ where $\left.\omega\right|_{X}$ is non-degenerate. Then $X_{\text {herm }}$ is a $\mathbf{D}$-Kähler manifold in the induced metric.

Consider now a $\mathbf{D}$-submanifold

$$
V(\mathbf{D}) \subset \mathbf{P}^{n}(\mathbf{D})
$$

of $\mathbf{D}$-dimension $k$ arising from an algebraic submanifold

$$
V(\mathbf{R}) \subset \mathbf{P}^{n}(\mathbf{R})
$$

as above, and recall the canonical product structure (B.1). The embedding (B.2) induces a riemannian metric $g$ on $V(\mathbf{R})$ from the standard spherical metric $g_{0}$ on $\mathbf{P}^{n}(\mathbf{R})$. The embedding (B.3) induces a Kähler metric with Kähler form $\omega$ on the subset $V(\mathbf{D})_{\text {herm. }}$. There is also a nowhere null holomorphic $k$-form

$$
\Phi=e \cdot \alpha+\bar{e} \cdot \beta
$$

where $\alpha$ and $\beta$ denote the volume form of $V(\mathbf{R})$, with metric $g_{0}$, pulled back from the first and second factors of (B.1) respectively. Note that

$$
\operatorname{Re}(\Phi)=\frac{1}{2}(\beta+\alpha) \quad \text { and } \quad \operatorname{Im}(\Phi)=\frac{1}{2}(\beta-\alpha) .
$$

The following pretty geometry is straightforward to verify.

Proposition B.4. Consider the diagonal embedding $\Delta: V(\mathbf{R}) \hookrightarrow V(\mathbf{D})$ given by $\Delta(u)=(u, u)$ with respect to (B.1). Set $V_{\Delta}=\Delta(V(\mathbf{R}))$. Then:

(1) $V_{\Delta} \subset V(\mathbf{D})_{\text {herm }}$,

(2) $V_{\Delta}$ is space-like Lagrangian, in particular

$$
\left.\omega\right|_{V_{\Delta}}=0
$$

(3) $V_{\Delta}$ satisfies the "special" condition

$$
\left.\operatorname{Im}(\Phi)\right|_{V_{\Delta}}=0
$$

(4) the restriction of the (real part of) the Kähler metric to $V_{\Delta}$ coincides with $2 g$,

(5) there is a constant $c=c(k)$ such that:

$$
\left.\operatorname{Re}(\Phi)\right|_{V_{\Delta}}=c \operatorname{vol}(g)
$$

The story is similar for the anti-diagonal embedding $\widetilde{\Delta}: V(\mathbf{R}) \hookrightarrow V(\mathbf{D})$ given by $\widetilde{\Delta}(u)=$ $(u,-u)$ except that $V_{\widetilde{\Delta}}$ is purely time-like instead of space-like, and the induced metric is $-2 g$. 
Of course the Kähler metric on $V(\mathbf{D})_{\text {herm }}$ is not Ricci-flat. However, it is interesting to ask whether the metric and the holomorphic $n$-form $\Phi$ can be altered outside $V_{\Delta}$ so that so that $\Phi$ becomes parallel.

\section{Appendix C}

\section{Degenerate Projections.}

Many of the results in Special Lagrangian geometry which appeared in $\left[\mathrm{HL}_{1}\right]$ carry over to the split case. One instance of this concerns submanifolds with degenerate projections. For what follows the reader is referred to $\left[\mathrm{HL}_{1}\right]$ for full details and proofs.

Decompose $\mathbf{R}^{n}$ as $\mathbf{R}^{n}=\mathbf{R}^{p} \oplus \mathbf{R}^{n-p}$ and consider a $p$-dimensional submanifold $M$ in $\mathbf{R}^{p} \oplus$ $\tau \mathbf{R}^{n-p}$ along with a smooth function $h$ on $M$. Let $N_{\text {twist }}(M)$ denote the normal bundle to $M$ twisted by the antipodal map on the fibres, defined precisely by (C.2) below. This is a Lagrangian submanifold of $\mathbf{D}^{n}$ as is its affine translate by $d h$,

$$
X \equiv N_{\text {twist }}(M)+d h .
$$

The case of interest is when $M$ is space-like in $\mathbf{R}^{p} \oplus \tau \mathbf{R}^{n-p}$. Then $M \equiv \operatorname{graph}(u)$ with $u: \mathbf{R}^{p} \rightarrow$ $\mathbf{R}^{n-p}$, and we can assume $h: \mathbf{R}^{p} \rightarrow \mathbf{R}$.

Using the decomposition of $\mathbf{R}^{n}$ to decompose the coordinates $z=\left(x^{\prime}, x^{\prime \prime}\right), y=\left(y^{\prime}, y^{\prime \prime}\right)$ the manifold $X$ is parameterized by sending $x \in \mathbf{R}^{n}$ to

$$
\left(x^{\prime}, x^{\prime \prime}, x^{\prime \prime} \cdot \frac{\partial u}{\partial x^{\prime}}\left(x^{\prime}\right)+\frac{\partial h}{\partial x^{\prime}}\left(x^{\prime}\right), u\left(x^{\prime}\right)\right) .
$$

Therefore, $X$ is the graph (over $\mathbf{R}^{n}$ in $\mathbf{D}^{n}=\mathbf{R}^{n} \oplus \tau \mathbf{R}^{n}$ ) of $\nabla \varphi$, the gradient of the potential function

$$
\varphi(x) \equiv x^{\prime \prime} \cdot u\left(x^{\prime}\right)+h\left(x^{\prime}\right) .
$$

In the case $h \equiv 0$ we have:

THEOREM C.1. If $M$ is a space-like austere submanifold of $\mathbf{R}^{p} \oplus \tau \mathbf{R}^{n-p}$, then $N_{\text {twist }}(M)$ is a special Lagrangian submanifold of $\mathbf{D}^{n}$.

The case $n=3$ and $p=2$ is particularly nice even with $h \neq 0$. Note that $\varphi(x)=x_{3} u\left(x_{1}, x_{2}\right)+$ $h\left(x_{1}, x_{2}\right)$ is the potential function for $X$.

THEOREM C.2. The submanifold $X \equiv N_{\text {twist }}(M)+d h$ is special Lagrangian in $\mathbf{D}^{3}$ if and only if $M^{3}$ is space-like in $\mathbf{R}^{2} \oplus \tau \mathbf{R}$ with vanishing mean curvature and $h$ is a harmonic function on $M$.

Proof. If $\varphi(x)=x_{3} u\left(x_{1}, x_{2}\right)+h\left(x_{1}, x_{2}\right)$, then $\nabla \varphi=\left(x_{3} u_{1}+h_{1}, x_{3} u_{2}+h_{2}, u\right)$, and

$$
\operatorname{Hess} \varphi=\left(\begin{array}{ccc}
x_{3} u_{11}+h_{11} & x_{3} u_{12}+h_{12} & u_{1} \\
x_{3} u_{21}+h_{21} & x_{3} u_{22}+h_{22} & u_{2} \\
u_{1} & u_{2} & 0
\end{array}\right)
$$

Therefore

$$
\begin{aligned}
\Delta \varphi+\operatorname{detHess} \varphi=x_{3}[ & \left.\left(1-u_{2}^{2}\right) u_{11}+2 u_{1} u_{2} u_{12}+\left(1-u_{1}^{2}\right) u_{22}\right] \\
+ & {\left[\left(1-u_{2}^{2}\right) h_{11}+2 u_{1} u_{2} h_{12}+\left(1-u_{1}^{2}\right) h_{22}\right] . }
\end{aligned}
$$


Remark C.3. Each such special Lagrangian manifold $X \equiv N_{\text {twist }}(\operatorname{graph}(u))$ has a potential $\bar{\varphi}\left(u_{1}, u_{2}, u_{3}\right)$ with $X \equiv \operatorname{graph} \nabla \bar{\varphi}$ in $e \mathbf{R}^{3} \oplus \bar{e} \mathbf{R}^{3}$. The function $\bar{\varphi}$ is a solution to:

$$
\bar{\varphi} \text { convex and } \operatorname{detHess} \bar{\varphi}=1 \text {. }
$$

It would be interesting to examine some explicit examples such as $y_{3}=u\left(x_{1}, x_{2}\right)=\frac{1}{a} \arccos (\operatorname{ar}+$ c), $r=\sqrt{x_{1}^{2}+x_{2}^{2}}$

\section{Appendix D}

\section{Singularities and Semi-Riemannian Calibrations.}

It is straightforward to extend the basic set-up of Section 15 to manifolds. Suppose that $X$ is a semi-riemannian manifold of signature $p, q$ with $p, q \geq 1$. We identify the Grassmannian $G_{\text {space }}(p)_{x}$ of oriented space-like $p$-planes in $T_{x} X$ with a subset of $\Lambda^{p} T_{x} X$ by choosing an oriented orthonormal basis for each such $p$-plane and taking the wedge product. Since $p, q \geq 1$, each $G_{\text {space }}(p)_{x}$ has two connected components. A space-like orientation on $X$ is a continuous choice of one the components, denoted by $G_{\text {space }}^{+}(p)$, and referred to as the Grassmannian of positive (or "future") oriented space-like $p$-planes.

Definition D.1. The mass ball $B_{x} \subset \Lambda^{p} T_{x} X$ is the convex hull of $G_{\text {space }}^{+}(p)_{x}$. The comass ball $B_{x}^{*} \subset \Lambda^{p} T_{x}^{*} X$ is defined to be the polar:

$$
B_{x}^{*} \equiv\left\{\phi \in \Lambda^{p} T_{x}^{*} X: \phi(\xi) \geq 1 \text { for all } \xi \in G_{\text {space }}^{+}(p)_{x}\right\}
$$

(or equivalently for all $\xi \in B_{x}$ ). The mass cone $\mathcal{C}_{x}$ is the (convex) cone on $B_{x}$ with vertex at the origin in $\Lambda^{p} T_{x} X$, and the comass cone $\mathcal{C}_{x}^{*}$ is the (convex) cone on $B_{x}^{*}$ with vertex at the origin in $\Lambda^{p} T_{x}^{*} X$.

Note that the closure $\overline{\mathcal{C}_{x}^{*}}$ is the polar cone of $\overline{\mathcal{C}_{x}}$, i.e., $\phi \in \overline{\mathcal{C}_{x}^{*}} \Longleftrightarrow \phi(\xi) \geq 0$ for all $\xi \in \overline{\mathcal{C}_{x}}$. Given $\xi \in \mathcal{C}_{x}$, there exists a unique $\lambda>0$ such that $\lambda \xi \in \partial B_{x}$. We define

$$
\|\xi\| \equiv \frac{1}{\lambda} \quad \text { to be the mass norm of } \xi
$$

Similarly, the comass norm of $\phi \in \mathcal{C}_{x}^{*}$, denoted by $\|\phi\|^{*}$, is defined so that $\|\phi\|^{*}=1 \Longleftrightarrow$ $\phi \in \partial B_{x}^{*}$ and so that $\|\phi\|^{*}$ is homogeneous of degree 1 for positive scalars. (Note that in the analogous positive definite case the mass and comass cones are all of $\Lambda^{p} T_{x} X$ and $\Lambda^{p} T_{x}^{*} X$.) Both $\|\cdot\|$ and $\|\cdot\|^{*}$ are superadditive on $\mathcal{C}_{x}$ and $\mathcal{C}_{x}^{*}$ respectively.

Definition D.2. A $d$-closed $p$-form $\phi$ on $X$ which takes its values in the comass cone $\mathcal{C}$ is a calibration if it has comass one at each point, i.e.,

$$
\phi(\xi) \geq 1 \text { for all } \xi \in G_{\text {space }}^{+}(p) .
$$

A calibration $\phi$ is said to calibrate the set $G(\phi) \equiv\{\phi=1\} \cap G_{\text {space }}^{+}(p)$ of $p$-planes in $G_{\text {space }}^{+}(p)$ where $\phi$ attains its minimum. An oriented submanifold $M$ of $X$ is a $\phi$-submanifold if $\vec{M} \in G(\phi)$ at each point, or equivalently, $M$ is positive space-like and $\left.\phi\right|_{M}$ is the unit volume form for $M$.

The fundamental theorem for semi-riemannian calibrations (see Theorem 15.3 for the case $X=\mathbf{R}^{p, q}$ ) can be stated for submanifolds as follows. See the generalization allowing singularities, Theorem D.10 below, for the proof. 
Proposition D.3. Suppose $\phi$ is a calibration of degree $p$ on a semi-riemannian manifold with a space-like orientation an of signature $p, q$ with $p, q \geq 1$. If $M$ is a compact $\phi$-submanifold (possibly with boundary), then

$$
\operatorname{vol}(M) \geq \operatorname{vol}(N)
$$

for all positive space-like compact submanifolds $N$ which are homologous to $M$. Moreover, if equality occurs, then $N$ is also a $\phi$-submanifold.

The purpose of this appendix is to generalize this result to allow singularities.

\section{Currents of Special Type $\mathcal{S}$.}

A current $T$ of dimension $p$ on a manifold $X$ which is representable by integration (see $[\mathrm{F}]$ ) can be put in polar form in each coordinate system $U \subset \mathbf{R}^{n}$

$$
T=\vec{T}\|T\|
$$

where $\|T\|$ is a non-negative Radon measure called the generalized volume measure for $T$, and $\vec{T}$ is a $\|T\|$-measurable function taking values in the boundary of the unit mass ball in $\Lambda^{p} \mathbf{R}^{n}$, i.e., $|\vec{T}|=1\|T\|$-a.e.. It is referred to as the generalized tangent space $(\vec{T}(x)$ may not be simple). Of course

$$
T(\varphi)=\int \varphi(\vec{T})\|T\|
$$

for each test $p$-form $\varphi$ on $U$.

Each riemannian metric on $X$ determines a second notion of mass norm $|\cdot|^{\prime}$ on $\Lambda^{p} T_{x} X$ (with $x \in U)$. Defining

$$
\|T\|^{\prime} \equiv|\vec{T}|^{\prime}\|T\| \quad \text { and } \quad \vec{T}^{\prime}(x) \equiv \frac{1}{|\vec{T}(x)|^{\prime}} \vec{T}(x)
$$

yields the polar form

$$
T=\vec{T}^{\prime}\|T\|^{\prime}
$$

based on the riemannian metric. Here $\|T\|^{\prime}$ is a Radon measure on $X$ and ${\overrightarrow{T^{\prime}}}^{\prime}(x)$ is a $\|T\|^{\prime}$ measurable section of the bundle $\Lambda^{p} T X$ with unit mass norm almost everywhere.

Now given a riemannian metric we drop the prime from the notation and use $\vec{T}$ and $\|T\|$ based on the riemannian metric.

Suppose now that $\mathcal{S}$ is any cone set (i.e., $s \in \mathcal{S}_{x} \Rightarrow t s \in \mathcal{S}_{x} \forall t>0$ ) in the total space of the bundle $\Lambda^{p} T X$. Given a current $T$ of dimension $p$ which is representable by integration, if

$$
\vec{T}(x) \in \mathcal{S}_{x} \text { for }\|T\| \text { a.a. } x
$$

is true for one riemannian metric, then it is true for all riemannian metrics. Consequently, the following concept is independent of the choice of riemannian metric on $X$

Definition D.4. Suppose $\mathcal{S}$ is a cone set in $\Lambda^{p} T_{x} X$. A current $T$ of dimension $p$ which is representable by integration is said to be of type $\mathcal{S}$ if

$$
\vec{T}(x) \in \mathcal{S}_{x} \text { for }\|T\| \text { a.a. } x \text {. }
$$

\section{The Fundamental Theorem for Semi-Riemannian Calibrations $\mathcal{S}$.}


Suppose that $X$ is a semi-riemannian manifold of signature $p, q \geq 1$ which is space-like oriented and with mass cone $\mathcal{C} \subset \Lambda^{p} T X$.

Definition D.5. A current $T$ of dimension $p$ which is representable by integration is said to be positive space-like if $T$ is of type $\mathcal{C}$.

Proposition D.6. Each positive space-like current $T$ has a semi-riemannian polar form

$$
T=\vec{T}\|T\|
$$

where $\|T\|$ is a non-negative Radon measure on $X$ and $\vec{T}$ is a $\|T\|$-measurable section of $\Lambda^{p} T X$ with the semi-riemannian mass norm $|\vec{T}(x)|=1$ for $\|T\|$-a.a. points $x$.

Proof. Let $T=\vec{T}^{\prime}\|T\|^{\prime}$ denote a local riemannian polar form. Since $T$ is type $\mathcal{C}$, i.e., $\vec{T}^{\prime}(x) \in \mathcal{C}_{x}$, there is a well defined semi-riemannian mass norm $\left|\vec{T}^{\prime}(x)\right|$. Set

$$
\vec{T}(x) \equiv \frac{1}{|\vec{T}(x)|^{\prime}} \vec{T}^{\prime}(x) \quad \text { and } \quad\|T\| \equiv|\vec{T}|^{\prime}\|T\|^{\prime}
$$

Then $T=\vec{T}\|T\|$ and the semi-riemannian mass norm $|\vec{T}(x)|$ equals 1 for $\|T\|$-a.a. points $x$.

Definition D.7. Thevolume/mass of a positive space-like current $T$ is defined by

$$
\operatorname{vol}_{K}(T)=\int_{K}\|T\| \quad \text { for all compact } K \subset X
$$

using the generalized volume measure given by Proposition D.6.

Remark D.8. We leave it to the reader to show that this definition of mass for positive space-like currents $T$ agrees with the definition in [KMW]. Namely, $\int\|T\|$ equals the infimum of $T(\varphi)$ taken over all forms $\varphi$ of type $\mathcal{C}^{*}$ with comass $\|\varphi\|_{K}^{*} \equiv \inf _{x \in K}\|\varphi\|^{*} \geq 1$. (Hint: Use (15.3).)

Now suppose that $\phi$ is a calibration of degree $p$ on $X$.

Definition D.9. A $\phi$-subvariety is a locally rectifiable current $T$ of type $\mathcal{C}$, or equivalently of type $\mathbf{R}^{+} \cdot G_{\text {space }}^{+}(p)$ (the cone on $G_{\text {space }}^{+}(p)$ ). The $\phi$-subvariety is without boundary if $d T=0$.

The fundamental theorem of semi-riemannian calibrations can be stated a follows.

THEOREM D.10. Suppose $\phi$ is a calibration of degree $p$ on a space-like oriented, semiriemannian manifold of signature $p, q \geq 1$. Suppose that $T$ is a $\phi$-subvariety with compact support. Then $T$ is homologically volume maximizing. That is, for any other positive space-like rectifiable current $S$ with compact support which is homologous to $T(S=T+d R$ with $\operatorname{supp}(R)$ compact), one has

$$
\operatorname{vol}(T) \geq \operatorname{vol}(S)
$$

Moreover, equality occurs if and only if $S$ is also a $\phi$-subvariety.

Proof. Since $d \phi=0, S=T+d R$, and $\phi$ is a calibration, we have

$$
\operatorname{vol}(T)=\int\|T\|=\int \phi(\vec{T})\|T\|=T(\phi)=S(\phi)=\int \phi(\vec{S})\|S\| \geq \int\|S\|=\operatorname{vol}(S)
$$

and equality occurs if and only if $\phi(\vec{S})=1$ for $\|S\|$-a.a. points. 
Remark D.11. We leave it to the reader to extend this theorem to currents representable by integration but not necessarily rectifiable, which are of type $\mathcal{C}(\phi)$ where $\mathcal{C}(\phi)$ is the convex cone on $G(\phi)$.

Remark D.12. (Null Planes and the Mass Cone). Let $\overline{\mathcal{C}}$ be the closed convex cone on $G_{\text {space }}^{+}\left(p, \mathbf{R}^{p, q}\right)$. Assume for simplicity that $p \leq q$. Let $\mathcal{I}(p)$ denote the set of isometries $A: \mathbf{R}^{p} \rightarrow$ $\mathbf{R}^{q}$, i.e., $A^{t} A=I$, the identity on $\mathbf{R}^{p}$. Identify $\mathcal{I}(p)$ with a subset of $\Lambda^{p} \mathbf{R}^{p, q}$ by letting $\operatorname{graph}(A)$ denote the oriented graph of $A$ and setting $\xi_{A} \equiv\left(e_{1}+A e_{1}\right) \wedge \cdots \wedge\left(e_{p}+A e_{p}\right) \in \Lambda^{p} \mathbf{R}^{p, q}$. If $p=q=n$ we have the orthogonal group $\mathrm{O}(n) \subset \Lambda^{n} \mathbf{D}^{n}$.

Note that $\left\langle e_{i}+A e_{i}, e_{j}+A e_{j}\right\rangle=0$ for all $i, j$ since $A^{t} A=I$. That is $\xi_{A}$ is totally null.

Proposition D.13. The closed mass cone $\overline{\mathcal{C}} \subset \Lambda^{p} \mathbf{R}^{p, q}$ is the convex cone on $\mathcal{I}(p) \subset \Lambda^{p} \mathbf{R}^{p, q}$.

Proof. If $A \in \mathcal{I}(p)$, note that for $0 \leq t<1, \xi_{A}^{t}=\left(e_{1}+t A e_{1}\right) \wedge \cdots \wedge\left(e_{p}+t A e_{p}\right)$ rescaled by $\left(1-t^{2}\right)^{\frac{p}{2}}$ is an element of $G_{\text {space }}^{+}\left(p, \mathbf{R}^{p, q}\right)$. Hence $\mathcal{I}(p) \subset \overline{\mathcal{C}}$.

Conversely, if $\xi \in G_{\text {space }}^{+}\left(p, \mathbf{R}^{p, q}\right)$, then pick an oriented orthonormal basis $e_{1}, \ldots, e_{p}$ for $\xi$ and an orthonormal set $\bar{e}_{1}, \ldots, \bar{e}_{p}$ in $\mathbf{R}^{q}$. Define $n_{j}^{ \pm}=\frac{1}{2}\left(e_{j} \pm \bar{e}_{j}\right)$ so that $e_{j}=n_{j}^{+}+n_{j}^{-}$. Then $\xi=e_{1} \wedge \cdots \wedge e_{p}=\sum_{ \pm} n_{1}^{ \pm} \wedge \cdots \wedge n_{p}^{ \pm}$and each $2^{p} n_{1}^{ \pm} \wedge \cdots \wedge n_{p}^{ \pm} \in \mathcal{I}(p)$.

\section{Appendix E \\ Singularities in Split SLAG Geometry.}

Recall the definition of type (Definition D.4) and the semi-riemannian polar form (Proposition D.6) for currents representable by integration.

Definition E.1. A locally rectifiable $n$-current $T$ defined on an open subset of $\mathbf{D}^{n}$ is a split SLAG subvariety if

$$
\vec{T} \in \widetilde{\mathrm{SLAG}} \text { for }\|T\| \text { a.a. points, }
$$

and is positive space-like if

$$
\vec{T} \in G_{\text {space }}^{+}\left(n, \mathbf{D}^{n}\right) \text { for }\|T\| \text { a.a. points. }
$$

The inequality in Theorem 4.4 reads

$$
(\operatorname{Re} d z)\left(\vec{T}_{x}\right) \geq 1 \quad \text { for all } \vec{T}_{x} \in G_{\text {space }}^{+}\left(n, \mathbf{D}^{n}\right),
$$

with equality $\Longleftrightarrow \vec{T}_{x}$ is split special Lagrangian, i.e., $\vec{T}_{x} \in \widehat{\text { SLAG }}$.

Theorem 5.3 generalizes to include "singularities".

THEOREM E.2. Suppose that $T$ is a split $S L A G$ subvariety with compact support in $\mathbf{D}^{n}$. Suppose $S$ is a positive space-like compactly supported rectifiable current on $\mathbf{D}^{n}$ with $d S=d T$. Then

$$
\operatorname{vol}(T) \geq \operatorname{vol}(S)
$$

and equality holds if and only if $S$ is also a split SLAG subvariety.

Proof. This theorem is a special case of the fundamental Theorem D.10.

Finally, we note that if the space-like requirement on $\vec{T}$ is dropped, the currents $T$ are still of interest (see Appendix F on dimension two). 
Definition E.3. A locally rectifiable $n$-current $T$ defined on an open subset of $\mathbf{D}^{n}$ is unconstrained split SLAG (not necessarily space-like split SLAG) if

(1) $T \wedge \omega=0 \quad$ (i.e., $T$ is Lagrangian), and

(2) $T \wedge \psi=0 \quad$ where $\psi=\operatorname{Im} d z=\frac{1}{2}(d v-d u)$.

\section{Appendix F}

Split SLAG Singularities in Dimension Two.

This appendix is devoted to describing the structure in dimension $n=2$ of $d$-closed currents which are unconstrained split SLAG (cf. Definition D.4) and currents that are split SLAG varieties. We use the corresponding results for complex curves which are collected together as a remark.

Remark F.1. (Complex Curves in $\mathbf{C}^{2}$ ). Suppose $V$ is an irreducible complex curve defined near a point $z \in \mathbf{C}^{2}$. Even if $z$ is a singular point of $V$, the curve $V$ has a well-defined tangent line (with multiplicity $>1$ ). The current $[V]$ of integration over $V$ is well-defined and the singularities are isolated. The Gauss map is an open map which can be understood using a local uniformizing parameter. A holomorphic chain $T$ in an open subset $X$ of $\mathbf{C}^{2}$ is defined to be a locally finite sum of the form $\sum_{j} n_{j}\left[V_{j}\right]$ where each $n_{j} \in \mathbf{Z}$ and each $V_{j}$ is a closed one-dimensional subvariety of $X$. Thus, holomorphic chains and divisors on $X$ are equivalent concepts.

Locally rectifiable 2-currents $T$ for which $\vec{T}$ is a field of complex lines, are completely understood because of the following result.

THEOREM F.2. ([HS], $[\mathbf{S}])$. Suppose that $T$ is a $d$-closed rectifiable 2-current defined in an open subset $X$ of $\mathbf{C}^{2}$. If the (unoriented) tangent space $\vec{T}$ to $T$ is a complex line $\|T\|$-a.e., then $T$ is a holomorphic chain in $X$.

This result can be viewed as a very strong regularity result for "rectifiable" complex curves. Fortunately, it applies directly to split SLAG geometry.

THEOREM F.3. There exists a real orthogonal coordinate change $\mathbf{C}^{2} \leftrightarrow \mathbf{D}^{2}$, such that holomorphic chains are transformed to unconstrained split SLAG currents and vice-versa.

Proof. Let $z^{\prime} \in \mathbf{C}^{2}$ denote complex coordinates and $z \in \mathbf{D}^{2}$ denote $\mathbf{D}$-coordinates with $z_{j}^{\prime}=$ $x_{j}^{\prime}+i y_{j}^{\prime}$ and $z_{j}=x_{j}+\tau y_{j}$. Define a coordinate change $z_{1}^{\prime}=x_{1}+i x_{2}$ and $z_{2}^{\prime}=y_{1}+i y_{2}$ from $\mathbf{D}^{2}$ to $\mathbf{C}^{2}$. Then

$$
d z^{\prime}=\omega_{\mathbf{D}}+i \operatorname{Im} d z
$$

Also we have

$$
\omega_{\mathbf{C}}=d x_{1} d x_{2}-d y_{1} d y_{2} \quad \text { and } \quad \operatorname{Re} d z=d x_{1}^{\prime} d x_{2}^{\prime}-d y_{1}^{\prime} d y_{2}^{\prime} .
$$

Suppose that $T$ is an unconstrained split SLAG current. Then by definition and (F.1) we have $T \wedge d z^{\prime}=0$ for $\|T\|$-a.a. points. This condition $T \wedge d z^{\prime}=0$ is equivalent to $\vec{T}$ being a complex line. Hence by the Structure Theorem F.2, $T$ is a holomorphic chain with respect to the complex structure $J$ on $\mathbf{D}^{2}$ defined by

$$
J\left(\frac{\partial}{\partial x_{1}}\right)=\frac{\partial}{\partial x_{2}} \quad \text { and } \quad J\left(\frac{\partial}{\partial y_{2}}\right)=\frac{\partial}{\partial y_{1}}
$$

Note that the condition

$$
\left.\operatorname{Im} d z\right|_{\operatorname{graph} A}=0 \quad \text { for } A \in M_{2}(\mathbf{R})
$$


is that $\operatorname{tr} A=0$. Hence, $M=\operatorname{graph} A$ is unconstrained split SLAG $\Longleftrightarrow A$ is symmetric with trace zero. Thus

$$
A=\left(\begin{array}{cc}
a & b \\
b & -a
\end{array}\right)
$$

and the graph $y=A x$ becomes $z_{2}^{\prime}=(a+i b) z_{1}^{\prime}$ in the complex coordinates $z_{1}^{\prime}, z_{2}^{\prime}$ above.

Now $M \equiv \operatorname{graph} A$, with $A$ as above, is split SLAG $\Longleftrightarrow$ in addition $A^{t} A=\left(a^{2}+b^{2}\right) \cdot I<I$, i.e.,

$$
|a+i b|<1
$$

Definition F.4. A holomorphic chain $T=\sum_{j} n_{j}\left[V_{j}\right]$ in $\mathbf{C}^{2}$ satisfies the $45^{\circ}$ rule if if each tangent plane is of the form $z_{2}^{\prime}=\alpha z_{1}^{\prime}$ with $|\alpha|<1$ (including tangent planes at singular points).

Corollary F.5. Under the real orthogonal coordinate transformation given in Theorem F.3, a split SLAG variety $T$ corresponds to a holomorphic chain $T$ which satisfies the $45^{\circ}$ rule.

Proof. By the hypothesis on $T$ that $\vec{T} \in \widetilde{\mathrm{SLAG}}$ at $\|T\|$-a.a. points, we only know that $T$ is a holomorphic chain whose tangent plane satisfies the $45^{\circ}$ rule at $\|T\|$-a.a. points. However, since the Gauss map of each $V_{j}$ is open (even in neighborhoods of singular points), it follows that then tangent planes satisfy the $45^{\circ}$ rule at every point of the support of $T$.

Remark F.6. These results can be restated in null coordinates on $\mathbf{D}^{2}$ as follows. Suppose $T$ is a $d$-closed locally rectifiable current on an open subset $X$ of $\mathbf{D}^{n}$, with the property that for $\|T\|$-a.a. points in $X$, the unoriented tangent space of $T$ is the graph of some symmetric matrix $A>0$ with $\operatorname{det} A=1$ over $e \mathbf{R}^{2}$ in $\mathbf{D}^{2}=e \mathbf{R}^{2} \oplus \bar{e} \mathbf{R}^{2}$. Then $T$ is a locally finite sum of currents of integration $\varphi_{*}([\Delta])$ where $\varphi: \Delta \rightarrow \mathbf{D}^{2}$ maps $\zeta$ to $z(\zeta)$ and

$$
x_{1}(\zeta)+i x_{2}(\zeta) \quad \text { and } \quad y_{2}(\zeta)+i y_{1}(\zeta) \text { are holomorphic. }
$$

Conversely, if $T=\varphi_{*}([\Delta])$ where $\varphi$ satisfies (F.2), and if

$$
\left|\frac{\partial}{\partial \zeta}\left(x_{1}(\zeta)+i x_{2}(\zeta)\right)\right|<\left|\frac{\partial}{\partial \zeta}\left(y_{2}(\zeta)+i y_{1}(\zeta)\right)\right|,
$$

then, except for isolated singularities, $\varphi$ parameterizes a split SLAG submanifold of $X \subset \mathbf{D}^{2}$. 


\section{References}

$\left[\mathrm{A}_{1}\right]$ A. D. Alexandrov, Almost everywhere existence of the second differential of a convex function and properties of convex surfaces connected with it (in Russian), Lenningrad State Univ. Ann. Math. 37 (1939), 3-35.

$\left[\mathrm{A}_{2}\right]$ A. D. Alexandrov, The Dirichlet problem for the equation $\operatorname{Det}\left\|z_{i, j}\right\|=\psi\left(z_{1}, \ldots, z_{n}, x_{1}, \ldots, x_{n}\right)$, I. Vestnik, Leningrad Univ. 13 No. 1, (1958), 5-24.

[AMT] D. V. Alekseevsky, C. Medori and A. Tomassini, Homogeneous para-Káhler Einstein manifolds, Russian Math. Surveys 64, No. 1, (2009), 1-43. ArXiv:0806.2272.

[B] R. Bryant, Classical, exceptional, and exotic holonomies: a status report, Actes de la Table Ronde de Géométrie Différentielle (Luminy, 1992), 93-165, Sémin. Congr., 1, Soc. Math. France, Paris, 1996.

$\left[\mathrm{C}_{1}\right]$ L. A. Caffarelli, Interior a priori estimates for solutions of fully non-linear equations, Ann. of Math. 130 (1990), 189-213.

$\left[\mathrm{C}_{2}\right]$ L. A. Caffarelli, A localization property of viscosity solutions to the Monge-Ampère equation and their strict convexity, Ann. of Math. 131 (1990), 129-134.

$\left[\mathrm{C}_{3}\right]$ L. A. Caffarelli, Interior $W^{2, p}$ estimates for solutions of the Monge-Ampère equation, Ann. of Math. 131 (1990), 135-150.

$\left[\mathrm{CNS}_{1}\right]$ L. Caffarelli, L. Nirenberg and J. Spruck, The Dirichlet problem for nonlinear second order elliptic equations. I: Monge-Ampère equation, Comm. Pure Appl. Math. 37 (1984), 369-402.

$\left[\mathrm{CNS}_{2}\right]$ L. Caffarelli, L. Nirenberg and J. Spruck, The Dirichlet problem for nonlinear second order elliptic equations, III: Functions of the eigenvalues of the Hessian, Acta Math. 155 (1985), 261-301.

$\left[\mathrm{CNS}_{3}\right]$ L. Caffarelli, L. Nirenberg and J. Spruck, The Dirichlet problem for the degenerate MongeAmpère equation, Rev. Mat. Iberoamericana 2 (1986), 19-27.

$\left[\mathrm{CNS}_{4}\right]$ L. Caffarelli, L. Nirenberg and J. Spruck, Correction to: "The Dirichlet problem for nonlinear second order elliptic equations. I: Monge-Ampère equation", Comm. Pure Appl. Math. 40 (1987), 659-662.

[CIL] M. G. Crandall, H. Ishii and P. L. Lions User's guide to viscosity solutions of second order partial differential equations, Bull. Amer. Math. Soc. (N. S.) 27 (1992), 1-67.

[CFG] V. Cruceanu, P. Fortuny and P. Gadea, A survey on paracomplex geometry, Rocky Mountain J. Math. 26 (1996), 83-115.

[D] Y. Dong, On indefinite special Lagrangian submanifolds in indefinite complex Euclidean spaces, Journal of Geometry and Physics 59 (2009), 710-726.

[EST] F. Etayo, R. Santamaría amd U. Trías The geometry of a bi-lagrangian manifold, Diff. Geom. Appl. bf 24 (2006). 33-59.

[Fu] L. Fu, On the boundaries of Special Lagrangian submanifolds, Duke Math. J. 79 no. 2 (1995), 405-422.

[GM] P. Gadea and A. Montesinos Amilibia, Spaces of constant para-holomorphic sectional curvature, Pacific J. Math. 136 (1989), 85-101.

[GGM] P. Gadea, J. Grifone and J. Muñoz Masqué, Manifolds modelled over free modules over the double numbers, Acta. Math. Hungar. 100 (2003). 187-203.

[H] F. R. Harvey, Spinors and Calibrations, Perspectives in Math. vol.9, Academic Press, Boston, 1990 . 
$\left[\mathrm{HL}_{1}\right]$ F. R. Harvey and H. B. Lawson, Jr, Calibrated geometries, Acta Mathematica 148 (1982), 47-157.

$\left[\mathrm{HL}_{2}\right]$ F. R. Harvey and H. B. Lawson, Jr., An introduction to potential theory in calibrated geometry, Amer. J. Math. 131 no. 4 (2009), 893-944. ArXiv:math.DG/0710.3920.

$\left[\mathrm{HL}_{3}\right]$ F. R. Harvey and H. B. Lawson, Jr., Duality of positive currents and plurisubharmonic functions in calibrated geometry, Amer. J. Math. 131 no. 5 (2009), 1211-1240. ArXiv:math.DG/0804.1316.

$\left[\mathrm{HL}_{4}\right]$ F. R. Harvey and H. B. Lawson, Jr., Dirichlet duality and the non-linear Dirichlet problem, Comm. on Pure and Applied Math. 62 (2009), 396-443.

[HL $\left.{ }_{5}\right]$ F. R. Harvey and H. B. Lawson, Jr., Plurisubharmonicity in a general geometric context, Stony Brook Preprint. ArXiv 0804.1316.

$\left[\mathrm{HL}_{6}\right]$ F. R. Harvey and H. B. Lawson, Jr., Lagrangian plurisubharmonicity and convexity, Stony Brook Preprint (2007).

[HS] F.R. Harvey and B. Shiffman, A characterization of holomorphic chains, Ann. of Math., 99 (1974), 553-587.

[Ha $]$ M. Haskins, Constructing special lagrangian cones, Ph.D. Thesis, Univ. of Texas at Austin, 2000 .

[Ha 2$]$ M. Haskins, Special lagrangian cones, Amer. J. Math 126 (2004), 845-871.

$\left[\mathrm{Ha}_{3}\right]$ M. Haskins, The geometric complexity of Special lagrangian $T^{2}$-cones, Invent. Math. 157 (2004), 11-70.

$\left[\mathrm{HK}_{1}\right]$ M. Haskins and N. Kapouleas, Special lagrangian cones with higher genus links, Invent. Math. 167 (2007), 223-294.

$\left[\mathrm{HK}_{2}\right]$ M. Haskins and N. Kapouleas, Gluing constructions of Special lagrangian cones, to appear in the "Handbook of Geometric Analysis"

$\left[\mathrm{Hi}_{1}\right]$ N. Hitchin, The moduli space of special Lagrangian submanifolds, Ann. Scuola Norm. Sup. Pisa Cl. Sci. (4) 25 (1997), 503-515.

$\left[\mathrm{Hi}_{2}\right]$ N. Hitchin, Generalized Calabi-Yau manifolds, Quart. J. Math. 54 (2003), 281-308.

[IZ] S. Ivanov and S. Zamkovoy, Parahermitian and paraquaternionic manifolds, Diff. Geom. and its Applications 23 (2005), 205-234.

$\left[J_{1}\right]$ D. Joyce, pp. 163-198 in "Different Faces of Geometry", Int. Math. Ser. (N.Y.), 3, Kluwer/Plenum, New York, 2004.

$\left[\mathrm{J}_{2}\right]$ D. Joyce, U(1)-Invariant special Lagrangian 3-folds. III. Properties of singular solutions, Adv. Math. 192 (2005), 135-182.

[KM] Y.-H. Kim and R. J. McCann, Continuty, curvature, and the general covariance of optimal transportation, ArXiv:0712.3077. To appear in J. Eur. Math. Soc..

[KMW] Y.-H. Kim, R. J. McCann and M. Warren, Calibrating optimal transportation: a new pseudoriemannian geometry, ArXiv:0907.4962

$\left[\mathrm{Mc}_{1}\right]$ R. C. McLean, Deformations of Calibrated Submanifolds, Duke University Thesis, 1996.

$\left[\mathrm{Mc}_{2}\right]$ R. C. McLean, Deformations of Calibrated Submanifolds, Comm. Anal. Geom. 6 No. 4 (1998), 705-747.

$\left[\mathrm{M}_{1}\right]$ J. Mealy, Calibrations on semi-riemannian manifolds, Ph.D. Thesis, Rice University, 1989.

$\left[\mathrm{M}_{2}\right]$ J. Mealy, Volume maximization in semi-riemannian manifolds, Indiana Univ. Math. J. 40 (1991), 793-814.

$\left[\mathrm{P}_{1}\right]$ A. V. Pogorelov, Monge-Ampère equations of elliptic type, Noordhoff, Groningen, 1964. 
$\left[\mathrm{P}_{2}\right]$ A. V. Pogorelov, Extrinsic geometry of convex surfaces, Translations A. M. S., Vol XXXV, 1973.

[RT] J. B. Rauch and B. A. Taylor, The Dirichlet problem for the multidimensional Monge-Ampère equation, Rocky Mountain J. Math 7 (1977), 345-364.

[SS] L. Schäfer and F. Schulte-Hengesbach, Nearly pseudo-Kähler and nearly para-Kähler six manifolds, ArXiv:0912.3271

[S] B. Shiffman, Complete characterization of holomorphic chains of codimension one, Math. Ann. 274 (1986), 233-256.

[TU] N. S. Trudinger and J.n I. E. Urbas, Second derivative estimates for equations of MongeAmpère type, Bull. Austral. Math. Soc. 30 (1984), 321-334.

[V] C. Villani, Optimal Transport: Old and New, Grundlehren der mathematischen Wissenscharften vol. 338, Srpinger, 2009.

$\left[\mathrm{W}_{1}\right]$ M. Warren, Calibrations associated to Monge-Ampere equations, ArXiv:0702291. To appear in Trans. A.M.S..

$\left[\mathrm{W}_{2}\right]$ M. Warren, A McLean theorem for the moduli space of Lie solutions to mass transport equations, ArXiv:1006.1334.

[Wo] J. Wood, Foliations on 3-manifolds, Annals of Math. 89 (1969), 336-358.

[Y] Yu Yuan, A priori estimates for solutions of fully nonlinear special lagrangian equations, Ann Inst. Henri Poincaré 18 (2001), 261-270.

F. Reese Harvey

Mathematics Department

Rice University

Houston, TX 77005-1892

e-mail: harvey@rice.edu

H. Blaine Lawson, Jr

Mathematics Department

Stony Brook University

Stony Brook, NY11794-3651

e-mail: blaine@math.sunysb.edu 